\title{
Ultra-High-energy Cosmic Rays from beyond the Greisen-Zatsepin-Kuz'min Horizon
}

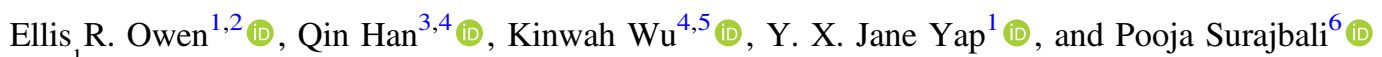 \\ ${ }^{1}$ Institute of Astronomy, National Tsing Hua University, Hsinchu, Taiwan, (ROC); erowen@ @app.nthu.edu.tw \\ ${ }^{2}$ Centre for Informatics and Computation in Astronomy, National Tsing Hua University, Hsinchu, Taiwan, (ROC) \\ ${ }^{3}$ School of Astronomy and Space Science, Nanjing University, 163 Xianlin Avenue, Nanjing, Jiangsu 210093, People's Republic of China \\ mg1826002@smail.nju.edu.cn \\ ${ }^{4}$ Mullard Space Science Laboratory, University College London, Holmbury St Mary, Surrey RH5 6NT, UK; kinwah.wu@ucl.ac.uk \\ ${ }^{5}$ Research Center for Astronomy, Astrophysics and Astrophotonics, Macquarie University, Sydney, NSW 2019, Australia \\ ${ }^{6}$ Max-Planck-Institut für Kernphysik, Saupfercheckweg 1, Heidelberg D-69117, Germany \\ Received 2021 January 12; revised 2021 July 25; accepted 2021 July 26; published 2021 November 16
}

\begin{abstract}
Ultra-high-energy (UHE) cosmic rays (CRs) of energies $\sim\left(10^{18}-10^{20}\right) \mathrm{eV}$, accelerated in violent astrophysical environments, interact with cosmic background radiation fields via photo-hadronic processes, leading to strong attenuation. Typically, the Universe would become "opaque" to UHE CRs after several tens of megaparsecs, setting the boundary of the Greisen-Zatsepin-Kuz'min (GZK) horizon. In this work, we investigate the contribution of sources beyond the conventional GZK horizon to the UHE CR flux observed on Earth, when photospallation of the heavy nuclear CRs is taken into account. We demonstrate that this contribution is substantial, despite the strong attenuation of UHE CRs. A significant consequence is the emergence of an isotropic background component in the observed flux of UHE CRs, coexisting with the anisotropic foreground component that is associated with nearby sources. Multi-particle CR horizons, which evolve over redshift, are determined by the CR nuclear composition. Thus, they are dependent on the source populations and source evolutionary histories.
\end{abstract}

Unified Astronomy Thesaurus concepts: Cosmic ray sources (328); Ultra-high-energy cosmic radiation (1733); Secondary cosmic rays (1438); Astronomical radiation sources (89); Extragalactic astronomy (506)

\section{Introduction}

Ultra-high-energy (UHE) ${ }^{7}$ cosmic-ray $(\mathrm{CR})$ events are rare. Although the detection of UHE CRs with energies $E>10^{20} \mathrm{eV}$ has been reported (e.g., Bird et al. 1994, 1995), their flux $J(E)$ at sea level, given by $E^{3} J(E) \approx 10^{24} \mathrm{eV}^{2} \mathrm{~m}^{-2} \mathrm{~s}^{-1} \mathrm{sr}^{-1}$, at energy $E=10^{20} \mathrm{eV}$ (see, e.g., Abu-Zayyad et al. 2013; Ivanov 2017), implies an event rate of only 1 particle per square kilometer per century. Several origins of these UHE CRs have been proposed, based on the argument that charged particles can, in principle, be accelerated to energies as high as $10^{20} \mathrm{eV}$ in astrophysical environments, including the relativistic jets of active galactic nuclei (AGN), the large-scale shocks associated with galaxy clusters, and neutron stars, as indicated by the Hillas (1984) criteria.

High-energy CR protons and nuclei interact with photons, and these interactions lead to the generation of lower energy subatomic particles. Cosmic Microwave Background (CMB) radiation and Extragalactic Background Light (EBL) permeate the Universe, and these provide a target for UHE CR interactions, via photo-hadronic processes. Such photo-hadronic interactions attenuate the flux of CRs at high energies, setting an energy limit above which the CR flux detected at Earth would drop very substantially. This energy is around $\sim 10^{20} \mathrm{eV}$ (Greisen 1966; Zatsepin \& Kuz'min 1966) and is known as the Greisen-Zatsepin-Kuz'min (GZK) limit (For reviews of GZK limit, see, e.g., Watson 2014; Kachelrieß \& Semikoz 2019). Photo-hadronic interactions also limit the intergalactic distance over which CR protons and nuclei can travel. For instance, the expected survival distance of a $\mathrm{CR}$ proton of $E_{\mathrm{p}} \approx 3 \times 10^{20} \mathrm{eV}$ traversing through a blackbody radiation field of a temperature $2.7 \mathrm{~K}$ ), is $<30 \mathrm{Mpc}$ (see, e.g.,

\footnotetext{
7 Kachelrieß \& Semikoz (2019) referred to cosmic rays with energies above $10^{17} \mathrm{eV}$ as UHE cosmic rays. The same terminology is adopted in this work.
}

Kachelrieß \& Semikoz 2019). This has led to a belief that the extragalactic UHE CR flux with $E \sim 10^{20} \mathrm{eV}$ detected on Earth is likely comprised of CRs originating in nearby foreground sources within a horizon (hereafter, referred to as the "GZK horizon") of radius $\sim 50 \mathrm{Mpc}$, and a residual, heavily attenuated background flux from more distant source populations. ${ }^{8}$

If we accept the Hillas (1984) criteria strictly, the number of possible UHE CR sources within the GZK horizon is limited. Because of this and the close proximity of sources, if the propagation of UHE protons and nuclei is ballistic ${ }^{9}$, their arrival directions as observed at Earth would not be isotropic. Instead, they would be preferentially oriented toward nearby UHE CR sources that fall within the GZK horizon. Recent studies (for reviews, see Kotera \& Olinto 2011; Deligny et al. 2017; Alves Batista et al. 2019b; Rieger 2019) have searched for anisotropies in the arrival directions of UHE CRs (Abreu et al. 2010; Aab et al. 2014, 2015, 2018; Pierre Auger Collaboration et al. 2017; Abbasi et al. 2018), with Pierre Auger Collaboration et al. (2017) reporting a detection for CRs of energies above $8 \times 10^{18} \mathrm{eV}$ at a significance $>5.2 \sigma$ (see section 4.2 for further discussion). Complementary studies investigating correlations between UHE CR arrival directions and catalogs of possible sources (in particular, AGN) have also returned encouraging results (see Pierre Auger Collaboration et al. 2007; Abreu et al. 2010). However, stronger correlations

\footnotetext{
8 Contributions from source populations beyond $\sim 50 \mathrm{Mpc}$ were considered in Berezinsky \& Grigor'eva (1988), where UHE CR flux from sources up to $z \sim 1.17$ were shown to provide a nonnegligible component of the detected flux; see also Kalashev et al. (2008) for more recent work considering the contribution from sources up to a few hundred megaparsecs.

9 The propagation of charged UHE CRs is influenced by the strength and structure of the magnetic fields in intergalactic space. CRs of higher energy $E$ and/or lower charge $Z$ (i.e. with higher rigidity $R=E / Z$ ) are deflected less. However, CRs with energies $>2 \times 10^{19} \mathrm{eV}$ would experience little deflection $\left(\leqslant 10^{\circ}\right)$ over $\sim 100 \mathrm{Mpc}$ (Alves Batista et al. 2019b).
} 
result if the UHE CR flux arriving at Earth is considered to have two components: an anisotropic source component (presumably from nearby high-energy cosmic accelerators), and an isotropic background component (Kim \& Kim 2011).

In this work, we investigate the interactions of UHE CRs with the CMB and EBL, considering in particular how photospallation and photopion interactions affect the propagation and attenuation of UHE CR nuclei. We investigate how much of the UHE CR flux originates from distant sources, and assess its dependence on CR composition and source population models. We show that distant CR sources, together with secondary CRs produced by photospallation could supply a significant isotropic flux in the UHE CRs observed at Earth. The paper is organized as follows: in Section 2, we introduce our models for the propagation and interaction of energetic CR nuclei through intergalactic radiation fields, accounting for the relevant microphysics and particle interactions. In Section 3, we introduce our CR source population, composition, and spectral model. We present our results in Section 4 and summarize our findings and present conclusions in Section 5.

\section{Cosmological Interactions and Propagation of Cosmic Rays}

\subsection{Ultra-High-energy Cosmic-Ray Interactions}

\subsubsection{Protons and Neutrons}

Energetic protons interact with ambient radiation fields to produce leptons and pions. These photo-hadronic interactions are dominated by Bethe-Heitler (BH) photo-pair production (Bethe \& Heitler 1934) and photopion production processes. Photo-pair processes proceed as

$$
A^{\prime}+\gamma \rightarrow A+l^{+}+l^{-},
$$

where $A^{\prime}$ and $A$ are nucleons before and after the interaction respectively, while $l^{+}$and $l^{-}$are the leptons and anti-leptons produced in this process. As photoelectron pair production contributes most to photo-pair CR energy losses (see e.g., Blumenthal 1970; Klein 2006) over the range of energies of interest in this work, ${ }^{10}$ we consider only the production of electrons $\left(e^{-}\right)$and positrons $\left(e^{+}\right)$. Without loss of generality, hereafter, we do not distinguish between electrons and positrons unless otherwise stated, and both are referred to as "electrons."

Photopion production operates mostly through the following channels: (i) resonant single-pion production, (ii) direct singlepion production, and (iii) multiple-pion production (Mücke et al. 1999). ${ }^{11}$ Resonant single-pion production arises through the production of $\Delta^{+}$intermediaries, which decay predominantly through the channels:

$$
p+\gamma \rightarrow \Delta^{+} \rightarrow\left\{\begin{aligned}
p+\pi^{0} \rightarrow p+2 \gamma \\
n+\pi^{+} \rightarrow n+\mu^{+}+\nu_{\mu} \\
\left\llcorner e^{+}+\nu_{\mathrm{e}}+\bar{\nu}_{\mu}\right.
\end{aligned}\right.
$$

(see Berezinsky \& Gazizov 1993). The decays produce charged and neutral pions, and their branching ratios (BRs) are roughly

\footnotetext{
${ }^{10}$ See Stepney \& Guilbert (1983) for an analytical fit for the effective crosssection of this process, which we adopt in our calculations.

11 Processes such as diffractive scattering (among others) may also operate, but are less important and do not arise at a rate comparable to those of the dominant channels.
}

$2: 3$ and 1:3, respectively. The pions will further decay, producing cascades of lower energy particles. Multi-pion production occurs at higher energies (see Mücke et al. 1999), and when the invariant interaction energy exceeds $\epsilon_{\mathrm{r}} \approx 3500$, charged and neutral pion production can arise with high multiplicities. For the energy range of interest in this work, each type of pion is produced in roughly equal proportion when all processes and the efficiencies of their production channels are taken into account (see Dermer \& Menon 2009).

Calculations for interaction lengths and their corresponding path lengths in blackbody intergalactic radiation fields were presented in Owen et al. (2018) and Owen et al. (2019). The same treatment is also adopted in this work. We note that free neutrons are produced in photo-hadronic interactions, as the one described in Equation (2), and these neutrons will undergo a $\beta^{-}$-decay:

$$
n \rightarrow p+e^{-}+\bar{\nu}_{\mathrm{e}}
$$

with a mean life of $880 \mathrm{~s}$ (Nakamura 2010). A neutron will decay into a proton, an electron, and an electron anti-neutrino if it does not collide and interact with other particles. In astrophysical environments, before $\beta^{-}$-decay occurs, a neutron could interact with the radiation field, leading to pion production:

$$
n+\gamma \rightarrow \Delta^{0} \rightarrow\left\{\begin{array}{l}
p+\pi^{-} \\
n+\pi^{0}
\end{array}\right.
$$

The BRs for $\Delta^{0} \rightarrow \pi^{-}$and $\Delta^{0} \rightarrow \pi^{0}$ are $1: 3$ and $2: 3$, respectively, near the energy threshold of the interaction (Hümmer et al. 2010).

The rates of photopion interactions for neutrons and protons do not differ much. Thus, the interaction cross-section and path length for high-energy neutrons can be approximated by the corresponding values for protons. (Hümmer et al. 2010; Romero \& Gutiérrez 2020). ${ }^{12}$ Despite the short mean life for neutron decay, neutrons can still traverse a long distance to collide with another particle or a photon because of time dilation resulting from their relativistic motion. However, in this work, we seek to assess the propagation distances and spectral form of UHE CRs based on their nucleon number $A$, with their charge only being consequential to specify the injection spectrum of each species. We assume that the injection of $A=1$ UHE CRs is dominated by protons, while CR neutrons only emerge as the interaction products of primary CRs. As such, we model the interactions and propagation of UHE CR neutrons and protons in the same way and do not distinguish between the two species. ${ }^{13}$

There are several consequences of the photopion processes. An isotropic cosmogenic neutrino component has been attributed to decaying pions arising from photopion interactions of CRs with intergalactic radiation fields (Allard et al. 2006; Rodrigues et al. 2021). Moreover, electrons produced in a

\footnotetext{
${ }^{12}$ The total interaction cross-sections for protons and neutrons only begin to differ substantially at much lower energies than of interest here, below $140 \mathrm{MeV}$ (Morejon et al. 2019). At low energies, Thomson scattering and pair production is possible for protons but is much weaker for neutrons (Gould 1993).

13 This would only differ in the presence of large-scale intergalactic magnetic fields which would deflect and modify the propagation of relativistic protons, but not neutrons. Such effects are not expected to substantially affect our results, and these details are left to dedicated future studies.
} 
photopion cascade, together with those electrons formed by $\mathrm{BH}$ pair production, could up-scatter the ambient low-energy photons to $\gamma$-rays, producing a diffuse $\gamma$-ray flux. In comparison with the $\gamma$-rays produced directly from the decay of neutral pions (a product of the photopion process), this Compton-scattered component is expected to be substantially weaker. ${ }^{14}$

\subsubsection{Nuclei}

$\mathrm{CR}$ nuclei interact with radiation fields via $\mathrm{BH}$ pair production, (nuclear) photopion production, and nuclear photospallation. $\mathrm{BH}$ pair production proceeds as

$$
N_{1}^{\prime}+\gamma \rightarrow N_{1}+l^{+}+l^{-}
$$

(Blumenthal 1970), where $N_{1}{ }^{\prime}$ and $N_{1}$ are the nuclei before and after the interaction, respectively. The $\mathrm{BH}$ process is $Z^{2}$ times faster for nuclei with charge number $Z$ than for protons. Photopion production channels are the same for nuclei as they are for protons (see Section 2.1.1), with corresponding interaction products: for example, nuclear photopion processes can also contribute to cosmogenic neutrino production. However, during their interactions, higher kinetic energies are available for nuclei compared to that for protons. The contribution of protons is typically weighted by a factor of $A=m_{\mathrm{N}_{\mathrm{l}}} / m_{p}$ (e.g., see Stecker 1979; Dermer \& Menon 2009), which implies that heavier nuclei would lose more energy during photopion interactions than lighter nuclei.

The main nuclear photospallation channels are

$$
N_{1} \rightarrow\left\{\begin{array}{l}
N_{2}+p \\
N_{3}+2 p
\end{array}\right.
$$

where $N_{1}$ is the primary nucleus of an atomic mass $A$, and $N_{2}$ and $N_{3}$ are the secondary nuclei of atomic masses $A-1$ and $A-2$, respectively (Stecker 1969; Puget et al. 1976; Stecker \& Salamon 1999; Ahlers \& Salvado 2011). Note that neutrons can also be emitted instead of protons in this process. Other branches, which remove more than one or two nucleons, may occur in a single interaction. These processes, however, occur with much lower probability and are not included in our calculations.

Photospallation (disintegration) is dominated by the formation of the Giant Dipole Resonance (GDR) intermediary. The process may be considered as a two-step mechanism (Puget et al. 1976), where the first stage involves the photoabsorption by a nucleus and the subsequent stage is the emission of a single or multiple nucleons from the nucleus via a (statistical) decay (Hayward 1960). Supposing that each of these emitted nuclei retain a fraction of $1 / A$ of the primary nucleus in a photospallation event, an approximate relation can be obtained

\footnotetext{
${ }^{14}$ Note that Compton-scattered $\gamma$-ray cascades can be substantial when initiated by high-energy $\gamma$-rays. In these cascades, energetic $\gamma$-ray photons initiate pair production in low-energy cosmological radiation fields as they propagate, with the electron pairs Compton up-scattering ambient photons back to $\gamma$-ray energies, leading to a reprocessing effect on high-energy $\gamma$ radiation (e.g., Domínguez et al. 2011; Gilmore et al. 2012; Inoue et al. 2013; Owen et al. 2021). This is a separate process from the production of diffuse $\gamma$-rays by electrons formed in the photopion cascade, which are referred to instead as cosmogenic.
}

from the energy partition between nucleons as

$$
\epsilon_{A-1} \approx\left(\frac{A-1}{A}\right) \epsilon_{A}
$$

such that the energy of the produced secondary nuclei can be estimated in an interaction (see Dermer \& Menon 2009). Here, $\epsilon_{\mathrm{A}}$ and $\epsilon_{\mathrm{A}-1}$ are, respectively, the dimensionless energies of the nuclei before and after a photospallation event, with $\epsilon_{\mathrm{A}}=E_{\mathrm{A}} / m_{\mathrm{e}} c^{2}=\gamma_{\mathrm{A}}\left(m_{\mathrm{A}} / m_{\mathrm{e}}\right)$ and a similar expression for $\epsilon_{\mathrm{A}-1}$. A consequence of the photospallation of heavy nuclei is the production of lighter nuclei in large numbers. Each of these secondary nuclei interacts with ambient radiation, hence initiating a new chain of photospallation. UHE CR nucleons thus litter traces of particles along their paths when they propagate across the Universe.

\subsection{Cosmological Propagation of Energetic Particles}

The propagation of energetic particles in intergalactic space is described by the transport equation. In the regime appropriate for this study, it may take the following form:

$$
\frac{\partial \mathcal{N}_{A}}{\partial t}=-\nabla \cdot \mathcal{S}_{A}+\frac{\partial}{\partial \epsilon_{A}}\left[b \mathcal{N}_{A}\right]+\mathcal{Q}_{A}-\Lambda_{A} \mathcal{N}_{A}
$$

Here, $\epsilon_{A}$ is the energy (of the particles) and $b$ is the rate of energy loss (presumably caused by cooling). The index $A$ denotes the nuclear species. In this work, we relate nuclear charge to mass following the correspondence presented in Puget et al. (1976). The charge of a species is not strictly set unambiguously by its mass. However, in most cases, there is a clear relation between the mass number of a UHE CR and its species/charge, with alternative isotopes being unstable and not being preferentially formed. ${ }^{15}$ We further define $\mathcal{N}_{A}$ as the number density of the particles with mass number $A$, while $\mathcal{S}_{A}$ is the $\mathrm{CR}$ streaming term, which is given by $c \mathcal{N}_{A}$ in the observer's frame if diffusion is negligible (e.g., Webb \& Gleeson 1979). $\mathcal{Q}_{A}$ is the source term specifying the rate of particle injection. $\Lambda_{A} \mathcal{N}_{A}$ is the sink term specifying the rate of particle loss, with an efficiency set by the parameter $\Lambda_{A}$. In cosmological settings, we assign $\mathcal{N}_{A}$ as the co-moving number density of a particle species. Therefore, $\mathcal{N}_{A}=n_{A}(1+z)^{-3}$, where $n_{A}$ is the physical number density of a particle species, and $z$ is the cosmological redshift.

The streaming of UHE CRs is effectively the speed of light, c. Thus, Equation (8) becomes

$$
\frac{\partial n_{A}}{\partial t}=-c \frac{\partial n_{A}}{\partial s}+\frac{\partial}{\partial \epsilon_{A}}\left[b n_{A}\right]+(1+z)^{3} \mathcal{Q}_{A}-\Lambda_{A} n_{A},
$$

\footnotetext{
15 This is with the exception of $A=5$ and $A=8$, which do not form stable isotopes. Since the radioactive decay timescale is less than the one-nucleon photospallation timescale for all but ${ }^{53} \mathrm{Mn},{ }^{26} \mathrm{Al}$ and ${ }^{10} \mathrm{Be}$, we assume that the decay brings the secondary nucleus to the line of nuclear stability before the next photocollision (Stecker \& Salamon 1999). As such, we consider the abundances of nuclei with $A=5$ and $A=8$ to be zero, and that decays which would yield such products would instead undergo double nucleon photospallation to the next stable species in the decay chain.
} 
with $s$ as the co-moving distance. In terms of the cosmological redshift, this may be expressed as

$$
\begin{aligned}
\frac{\partial}{\partial t} n_{A}\left(\epsilon_{A}, z\right)= & -c\left(\frac{d s}{d z}\right)^{-1} \frac{\partial}{\partial z} n_{A}\left(\epsilon_{A}, z\right) \\
& +\frac{\partial}{\partial \epsilon_{A}}\left[b_{A}\left(\epsilon_{A}, z\right) n_{A}\left(\epsilon_{A}, z\right)\right] \\
& +Q_{A}\left(\epsilon_{A}, z\right)-\Lambda_{A} n_{A}\left(\epsilon_{A}, z\right),
\end{aligned}
$$

where $Q_{A}=\mathcal{Q}_{A}(1+z)^{3}$. In a Friedmann-Lemaitre-Robertson-Walker (FLRW) universe,

$$
\frac{d s}{d z}=\frac{c \mathcal{E}(z)}{H_{0}(1+z)}
$$

and

$$
\mathcal{E}(z)=\left[\Omega_{\mathrm{r}, 0}(1+z)^{4}+\Omega_{\mathrm{m}, 0}(1+z)^{3}+\Omega_{\Lambda, 0}\right]^{-1 / 2}
$$

(see, e.g., Peacock 1999), where $\Omega_{\mathrm{m}, 0}=0.315 \pm 0.007$, $\Omega_{\mathrm{r}, 0} \approx 0$, and $\Omega_{\Lambda, 0}=0.685 \pm 0.007$ are the normalized density parameters for matter, radiation, and dark energy, respectively. The present value of the Hubble parameter $H_{0}=$ $100 h \mathrm{~km} \mathrm{~s}^{-1} \mathrm{Mpc}^{-1}$, where $h=0.673 \pm 0.006$ (Planck Collaboration et al. 2020).

A quasi-steady condition is adopted in our calculations, and this greatly simplifies the procedures involved in solving the particle-transport equations. The quasi-steady condition is justified, as the time of propagation of the particles across length-scales of the order of their horizons is much shorter than the Hubble time. Setting $\partial_{t}=0$, Equation (10) becomes

$$
\frac{\partial n_{A}}{\partial z}=\frac{1}{c}\left[\frac{\partial}{\partial \epsilon_{A}}\left(b_{A} n_{A}\right)+Q_{A}-\Lambda_{A} n_{A}\right] \frac{d s}{d z},
$$

which will be used in Section 2.2.1 for the transport calculations of $\mathrm{CR}$ protons and nuclei.

\subsubsection{Interaction Rates}

The source term in Equation (13) has two components,

$$
Q_{A}=Q^{\mathrm{a}}+Q^{\mathrm{sp}} .
$$

Here, $Q^{\mathrm{a}}$ specifies the rate primary nuclei are supplied by a source population of cosmic accelerators, and is defined by the source population, its evolution (specified by redshift, $z$ ), and the CR energy $\epsilon_{A}$ and mass number $A$ (our adopted models are discussed in Section 3). $Q^{\mathrm{sp}}$ is the rate of production of secondary nuclei from photospallation of heavy nuclei (i.e., the particle cascade contribution). We consider that the injected particles are represented by ${ }^{1} \mathrm{H},{ }^{4} \mathrm{He},{ }^{14} \mathrm{~N},{ }^{28} \mathrm{Si}$, and ${ }^{56} \mathrm{Fe}$ (with their relative abundance fractions detailed in Section 3). The spallation (disintegration) of heavy CR nuclei produces secondary nuclei with mass numbers ranging from 55 to 1 , and the injection rate of secondary nuclei (for mass number less than 56) by photospallation particle cascades is given by

$$
Q^{\mathrm{sp}}{ }_{A}\left(z, \epsilon_{A}\right)=\sum_{A^{\prime}} \int_{\epsilon_{\min }}^{\epsilon_{\max }} d \epsilon_{\mathrm{A}^{\prime}} \frac{R_{A}^{A^{\prime}} n_{A^{\prime}}}{(1+z)^{3}} .
$$

Here, $\epsilon_{\min }$ and $\epsilon_{\max }$ are the minimum and maximum energies considered in our calculations. We set the lowest energy to be $\epsilon_{\min } m_{\mathrm{e}} c^{2}=3.98 \times 10^{18} \mathrm{eV}$, as it is not clear if the cosmic-ray spectrum is dominated by extragalactic particles at lower energies than this (Giacinti et al. 2012; Aloisio et al. 2014). The maximum energy is set to be $\epsilon_{\max } m_{\mathrm{e}} c^{2}=3.16 \times 10^{20} \mathrm{eV}$, corresponding to the most energetic UHE CRs detected at Earth; see Bird et al. (1995). The summation in $Q^{\mathrm{sp}}{ }_{A}\left(z, \epsilon_{A}\right)$ accounts for the primary nuclei from $A^{\prime}$ up to $A=56$, where the photospallation injection rate for the most massive species under consideration (i.e., $Q^{\mathrm{sp}}{ }_{56}$ ) is zero. $Q^{\mathrm{sp}}{ }_{A}$ is dependent on $n_{A^{\prime}}$ (where $A^{\prime}>A$ ). As such, we solve Equation (13) from the CR nuclear species with largest $A(=56)$ to those with the smallest $A\left(=1\right.$ for protons/neutrons). $R_{A}^{A^{\prime}}\left(\epsilon_{A^{\prime}}, \epsilon_{A}, z\right)$ is the differential rate of the photospallation of species with mass number $A^{\prime}$ into species with mass number $A$, in the lab frame, in the presence of a soft photon field $n_{\mathrm{ph}}\left(\epsilon_{\mathrm{ph}}\right)$. If the radiation field is considered to be isotropic, this can be expressed as

$$
\begin{aligned}
R_{A}^{A^{\prime}}= & \frac{c}{2 \gamma_{A}^{2}} \int_{0}^{\infty} d \epsilon_{\mathrm{ph}}\left\{\frac{n_{\mathrm{ph}}\left(\epsilon_{\mathrm{ph}}\right)}{\epsilon_{\mathrm{ph}}^{2}}\right. \\
& \left.\times \int_{0}^{2 \gamma_{A^{\prime}} \mathrm{eph}_{\mathrm{ph}}} d \epsilon_{\mathrm{r}} \epsilon_{\mathrm{r}} \sigma_{A^{\prime} \rightarrow A}\left(\epsilon_{\mathrm{r}}\right)\right\}
\end{aligned}
$$

(see Dermer \& Menon 2009). Here, $\gamma_{A^{\prime}}$ is the Lorentz factor of the primary nucleus, $\sigma_{A^{\prime} \rightarrow A}\left(\epsilon_{\mathrm{r}}\right)$ is the photospallation crosssection of the primary nucleus of $A^{\prime}$ to produce a secondary nucleus of $A$, and $\epsilon_{\mathrm{r}}$ is the total invariant interaction energy (in a dimensionless form). For a blackbody radiation field ${ }^{16}$, the integrals in Equation (16) can be evaluated analytically. This simplifies the calculations for the region where the $\mathrm{CMB}$ dominates the radiation field, or the EBL if it is approximated as a summation of blackbody spectra (see Appendix).

In the two-step mechanism for photospallation, proper accounting for the physical processes in both steps are required in order to obtain an accurate value for the cross-section (Puget et al. 1976). For the case of single nucleon emission, the crosssection can, however, be adequately approximated by a Lorentzian function:

$$
\sigma_{1}\left(\epsilon_{\mathrm{r}}\right)=\frac{\sigma_{0, \mathrm{~A}} \epsilon_{\mathrm{r}}^{2} \Delta_{n}^{2}}{\left(\epsilon_{0}^{2}-\epsilon_{\mathrm{r}}^{2}\right)^{2}+\epsilon_{\mathrm{r}}^{2} \Delta_{n}^{2}}
$$

(see Karakula \& Tkaczyk 1993; Wang et al. 2008). Photospallation may result in multiple nucleon emission when $\epsilon_{\mathrm{r}}$ is above $30 \mathrm{MeV}$ or below the pion-production threshold $(\simeq 150 \mathrm{MeV})$, albeit with a much smaller cross-section and lower rate. A more complicated expression is available to describe this variation in the cross-section (see Puget et al. 1976), which is appropriate when the ambient radiation field has a very hard spectrum (see Wang et al. 2008). However, for GZK attenuation calculations, the ambient radiation fields which dominates the interactions are the CMB and EBL, for which the simplified expression for the cross-section is sufficient.

\footnotetext{
${ }^{16}$ The photon number density of a blackbody radiation field may be expressed as $n_{\mathrm{ph}}\left(\epsilon_{\mathrm{ph}}\right)=f\left(8 \pi / \lambda_{\mathrm{c}}{ }^{3}\right)\left\{\epsilon_{\mathrm{ph}}{ }^{2} /\left[\exp \left(\epsilon_{\mathrm{ph}} / \Theta\right)-1\right]\right\}$, where $\lambda_{\mathrm{c}}$ is the Compton wavelength of an electron and $\Theta=k_{\mathrm{B}} T / m_{\mathrm{e}} c^{2} . f=1$ for an unmodified blackbody radiation field, e.g., the $\mathrm{CMB}$, but $f<1$ in the case of a diluted radiation field (e.g., EBL components).
} 
For analytical tractability, this formulation can be simplified further by replacing the Lorentzian with a delta function:

$$
\sigma_{1}\left(\epsilon_{\mathrm{r}}\right)=\sigma_{0, \mathrm{~A}} \Delta_{n} \delta\left(\epsilon_{\mathrm{r}}-\epsilon_{0}\right)
$$

(Wang et al. 2008; Dermer \& Menon 2009). This still yields a photospallation rate correct to within an order of magnitude (Wang et al. 2008), which is sufficient for the purposes of our demonstrative model. In Equation (18), the width of the crosssection in energy $\Delta_{n}=8 \mathrm{MeV}$, is given by the energy bandwidth of the giant resonance, and the (dimensionless) energy at which the giant resonance cross-section peaks is given by $\epsilon_{0}=83.46 A^{-0.21}$ for $A>4$, and $\epsilon_{0}=1.81 A^{2.433}$ for $A \leqslant 4$ (Karakula \& Tkaczyk 1993). The maximum value of the cross-section is $\sigma_{0, \mathrm{~A}}=1.45 \times 10^{-27} A \mathrm{~cm}^{2}$ (Wang et al. 2008). Substitution of this cross-section, given in Equation (18), into Equation (16) yields

$$
\begin{aligned}
R_{1}= & -\frac{4 \pi f(z) c \sigma_{0, \mathrm{~A}} \Delta_{n} \epsilon_{0} \Theta(z)}{\gamma_{A}{ }^{2} \lambda_{c}{ }^{3}} \\
& \times \ln \left[1-\exp \left(\frac{-\epsilon_{0}}{2 \gamma_{A} \Theta(z)}\right)\right]
\end{aligned}
$$

(see also Dermer \& Menon 2009, for an expression derived under similar circumstances). Here, $\lambda_{\mathrm{c}}$ is the Compton wavelength of electron, $\Theta=k_{\mathrm{B}} T / m_{\mathrm{e}} c^{2}$ is the dimensionless temperature of the radiation field, and $f=1$ for a blackbody radiation field, e.g., the CMB, but $f(z)<1$ in the case of diluted blackbody radiation (e.g., redshift-dependent EBL components; see Appendix). This rate can also be used to compute the specific absorption rate of UHE CR nuclei due to photospallation. For this, we adopt the single-nucleon emission approximation of Wang et al. (2008), giving

$$
\Lambda_{A}^{\mathrm{sp}} \approx \frac{R_{1}}{A} \text {. }
$$

This forms a component of the sink (or absorption) term in the transport equation, i.e., Equation (13).

Adopting the single-nucleon approximation simplifies Equation (15) to

$$
Q_{A}^{\mathrm{sp}}\left(z, \epsilon_{A}\right) \approx \frac{1}{(1+z)^{3}} \int_{\epsilon_{\min }}^{\epsilon_{\max }} d \epsilon_{A^{\prime}} R_{1} n_{A^{\prime}}
$$

for nuclei with mass number $A>1$, and

$$
\begin{aligned}
Q^{\mathrm{sp}}{ }_{1}\left(z, \epsilon_{1}\right) \approx & \frac{1}{(1+z)^{3}}\left\{2 \int_{\epsilon_{\min }}^{\epsilon_{\max }} d \epsilon_{2} R_{1} n_{2}\right. \\
& \left.+\sum_{A^{\prime}} \int_{\epsilon_{\min }}^{\epsilon_{\max }} d \epsilon_{A^{\prime}} R_{1} n_{A^{\prime}}\right\}
\end{aligned}
$$

for $A=1$ nuclei. Here we do not distinguish between protons and neutrons (see Section 2.1.1), and the first term in Equation (22) accounts for the photosplitting of $A=2$ nuclei into two individual nuclei.

Nuclei can also be absorbed by photopion interactions. The expression of the interaction reaction rate in this case takes the same form as that given by Equation (16), but with the substitution of the pion-production cross-section, $\sigma^{A \pi}$. In this work, we also approximate this with a delta function

$$
\sigma^{A \pi}=\frac{\pi \sigma_{0} \Gamma_{\mathrm{res}} A}{2} \delta\left(\epsilon-\epsilon_{\mathrm{res}}\right)
$$

where $\Gamma_{\text {res }}$ is introduced as the resonance width. Adopting the same parameterization of $\epsilon_{\text {res }}$ and $\sigma_{0}$ as Unger et al. (2015) ${ }^{17}$ (see also, e.g., Moncada et al. 2017), we obtain $\epsilon_{\text {res }} \approx 665$, $\sigma_{0}=5.0 \times 10^{-28} \mathrm{~cm}^{2}$, and $\Gamma_{\text {res }}=150 \mathrm{MeV}$. By substituting Equation (23) into Equation (16), we obtain an analytical expression for the interaction rate:

$$
\begin{aligned}
R_{A}^{A \pi}= & \frac{4 \pi f c}{\gamma_{A}{ }^{2} \lambda_{c}{ }^{3}} \int_{0}^{\infty} d \epsilon_{\mathrm{ph}}\left\{\frac{1}{\exp \left[\epsilon_{\mathrm{ph}} / \Theta\right]-1}\right. \\
& \left.\times \int_{0}^{2 \gamma_{A} \epsilon_{\mathrm{ph}}} d \epsilon_{\mathrm{r}} \epsilon_{\mathrm{r}} \pi \sigma_{0} \frac{\Gamma_{\mathrm{res}}}{2} A \delta\left(\epsilon_{\mathrm{r}}-\epsilon_{\mathrm{res}}\right)\right\} \\
= & -\frac{2 \pi^{2} f c \sigma_{0} \Gamma_{\mathrm{res}} A \epsilon_{\mathrm{res}} \Theta}{\gamma_{A}{ }^{2} \lambda_{c}^{3}} \\
& \times \ln \left[1-\exp \left(\frac{-\epsilon_{\mathrm{res}}}{2 \gamma_{A} \Theta}\right)\right]
\end{aligned}
$$

where the last line expresses the rate in a similar form to that for photospallation, which is given in Equation (19). The corresponding specific absorption rate of CRs due to photopion interactions then follows simply as

$$
\Lambda_{A}^{A \pi}=\frac{R_{A \pi}^{A \pi}}{A}
$$

such that the overall sink term for nuclei, for use in Equation (13), may be written as

$$
\Lambda_{A}=\Lambda_{A}^{\mathrm{sp}}+\Lambda_{A}^{A \pi} .
$$

The other CR interactions described in Section 2.1 typically operate as continuous loss processes, or such that only a small fraction of the particle's energy is transferred in a single interaction. These can be modeled as cooling processes and the total cooling rate experienced by a UHE CR, in terms of the dimensionless energy of the nucleon (again, we retain our earlier convention where energies are in units of electron rest mass), therefore consists of three components:

$$
b=-d \epsilon / d t=b_{A}^{\gamma A}+b_{\mathrm{rad}}+b_{\mathrm{ad}},
$$

where $b_{A}^{\gamma A}$ is the cooling rate due to photo-pair production, $b_{\text {rad }}$ is the radiative cooling rate, and $b_{\text {ad }}$ is the adiabatic cooling rate of UHE CR nuclei as they propagate through an expanding cosmology.

In our calculations for photo-pair production, the invariant dimensionless interaction energy $\epsilon_{\mathrm{r}} \gtrsim 60$, so a fitted approximation for the inelastic cross-section of photo-pair losses is available (see Jost et al. 1950; Bethe \& Maximon 1954; Blumenthal 1970; Stepney \& Guilbert 1983):

$$
\sigma^{\gamma A}\left(\epsilon_{\mathrm{r}}\right) \approx\left\{\frac{7}{6 \pi} \alpha_{\mathrm{f}} \ln \left[\frac{\epsilon_{\mathrm{r}}}{k_{\gamma e}}\right]\right\} \sigma_{\mathrm{T}},
$$

where $\alpha_{\mathrm{f}}$ is the fine-structure constant, $\sigma_{\mathrm{T}}$ is the Thomson cross-section, and $k_{\gamma e}$ is a fitting constant, which we set as

\footnotetext{
17 This was based on the computed cross-section values in Kampert et al. (2013); Alves Batista et al. (2013) using tools provided online at https:// github.com/CRPropa/CRPropa3-data.
} 
2.0 (see Owen et al. 2018). The CR cooling rate due to photopair losses can then be expressed as

$$
\begin{aligned}
b_{A}^{\gamma A}= & -\frac{d \epsilon_{A}}{d t} \\
= & -\frac{f(z) Z_{A}^{2} c}{2 \gamma_{A} A} \int_{\gamma_{A}^{-1}}^{\infty} d \epsilon_{\mathrm{ph}}\left\{\frac{1}{\exp \left[\epsilon_{\mathrm{ph}} / \Theta(z)\right]-1}\right. \\
& \left.\times \int_{2}^{2 \gamma_{A} \epsilon_{\mathrm{ph}}} \frac{d \epsilon_{\mathrm{r}} \epsilon_{\mathrm{r}} \sigma^{\gamma A}\left(\epsilon_{\mathrm{r}}\right)}{\sqrt{1+2 \epsilon_{\mathrm{r}}}}\right\}
\end{aligned}
$$

(Dermer \& Menon 2009; Aloisio et al. 2013a), where the lower boundary value of the inner integral follows from at least one pair of electrons being formed by the process. As in Owen et al. (2019), we assume that the electron-positron pairs are produced in the zero-momentum frame of the interaction, and the interaction energy is completely dominated by the contribution from the interacting nucleus (see also Protheroe \& Johnson 1996; Dermer \& Menon 2009). This yields

$$
b^{\gamma A}{ }_{A}\left(\epsilon_{A}\right) \approx-\frac{f Z_{A}^{2} c \alpha_{\mathrm{f}} \sigma_{\mathrm{T}} \mathcal{F}_{\gamma e}(u)}{\lambda_{\mathrm{c}}{ }^{3} \gamma_{A}{ }^{2} A u^{5 / 2}},
$$

where the dimensionless variable $u=\left(\gamma_{A} \Theta\right)^{-1}$, and the function $\mathcal{F}_{\gamma e}(u)$ takes the same form as that in Owen et al. (2019).

Radiative cooling is dominated by Compton scattering with the ambient radiation field. It arises at a rate of

$$
b_{\mathrm{rad}}\left(\epsilon_{A}, z\right)=-\frac{4}{3} \frac{\sigma_{\mathrm{T}} U(z)}{m_{\mathrm{e}} c}\left[\frac{\epsilon_{A}{ }^{1 / 2} Z_{A}}{A}\left(\frac{m_{\mathrm{e}}}{m_{\mathrm{p}}}\right)\right]^{4}
$$

(see the expression given in Puget et al. 1976), where $U(z)$ is the energy density of the radiation field (at redshift $z$ ). Note that, here, $b_{\text {rad }}$ is dependent on the charge of the nuclei $Z_{A}$.

Adiabatic losses due to cosmological expansion occur at a rate

$$
b_{\text {ad }}\left(\epsilon_{A}, z\right)=-\epsilon_{A} H_{0} \mathcal{E}(z)
$$

(see Gould 1975; Berezinsky \& Grigor'eva 1988; Berezinsky et al. 2006), where $\mathcal{E}(z)$ is given by Equation (12).

We compare the relative importance of each of these cooling and interaction processes in Figure 1, where the effective path length of a UHE CR nucleus $\left(\ell_{A}\right)$ is defined as the characteristic distance over which the CR loses its energy. This can be estimated from the cooling rate $b$ using $\ell_{A}=c\left[|b| / \epsilon_{A}\right]^{-1}$. In Figure 1, we show the effective loss lengths for UHE CR nuclei $(A=1$ for protons, $A=4$ for ${ }^{4} \mathrm{He}$ nuclei, and $A=56$ for ${ }^{56} \mathrm{Fe}$ nuclei) in intergalactic conditions at $z=0$, with continuous losses due to pair production and adiabatic losses shown by the solid lines. These are compared to conditions at $z=3$ in Figure 2, where the background radiation fields are more intense (leading to faster photo-pair losses and higher photopion and photospallation rates, but lower adiabatic loss rates due to the relatively lower rate of cosmic expansion at $z=3$ compared to the present Universe). Radiative losses are inconsequential in all cases. For the purposes of comparison, we also estimate corresponding effective path lengths for UHE CRs due to processes that we regard as absorption in our calculations, where a substantial amount of energy is lost by the $\mathrm{CR}$ in a single interaction. These are
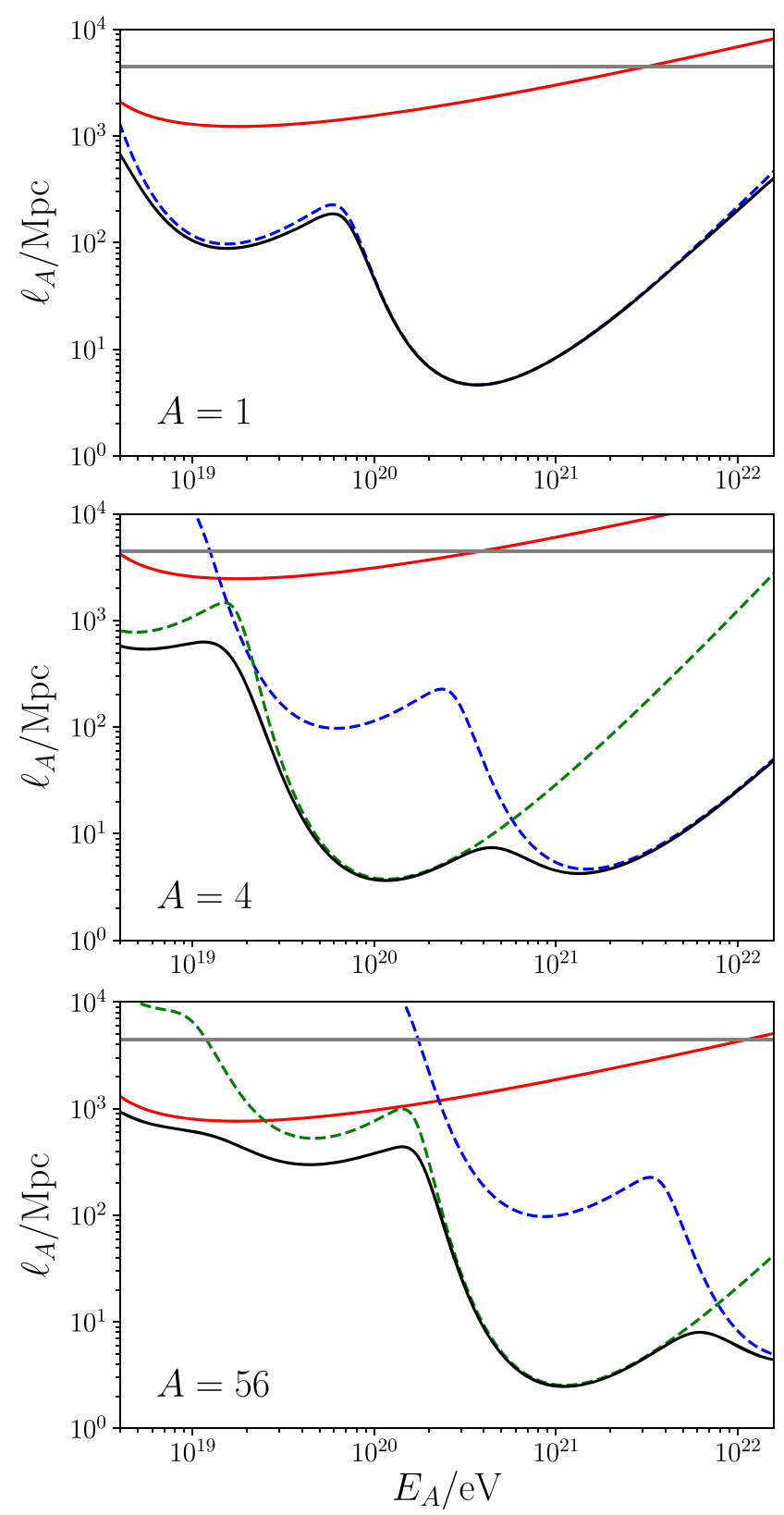

Figure 1. The characteristic lengths of energy loss for nuclei traversing through background (CMB and EBL) cosmological radiation fields at $z=0$, for various cooling and absorption processes. Panels from top to bottom correspond to protons $\left({ }^{1} \mathrm{H}\right)$, helium $\left({ }^{4} \mathrm{He}\right)$, and iron $\left({ }^{56} \mathrm{Fe}\right) \mathrm{CR}$ nuclei. Adiabatic losses due to cosmological expansion are represented by the gray lines; pair production by the red curves; photopion production by the blue dashed curves; and photospallation by the green dashed curves. Here, solid lines and curves denote continuous cooling loss, and dashed curves denote absorptive cooling loss. The solid black curves represent the total cooling.

photopion production and photospallation; note that photospallation only affects nuclei with $A>1$, and so protons $(A=1)$ are only affected by absorption through photopion interactions. These effective path lengths are distinguished as dashed lines in Figure 1, and are estimated by approximating these stochastic absorption process as a continuous loss process. This allows an equivalent cooling rate to be estimated as $b^{A \pi}{ }_{A} \approx-\epsilon_{A} \Lambda^{A \pi}{ }_{A}$ for 

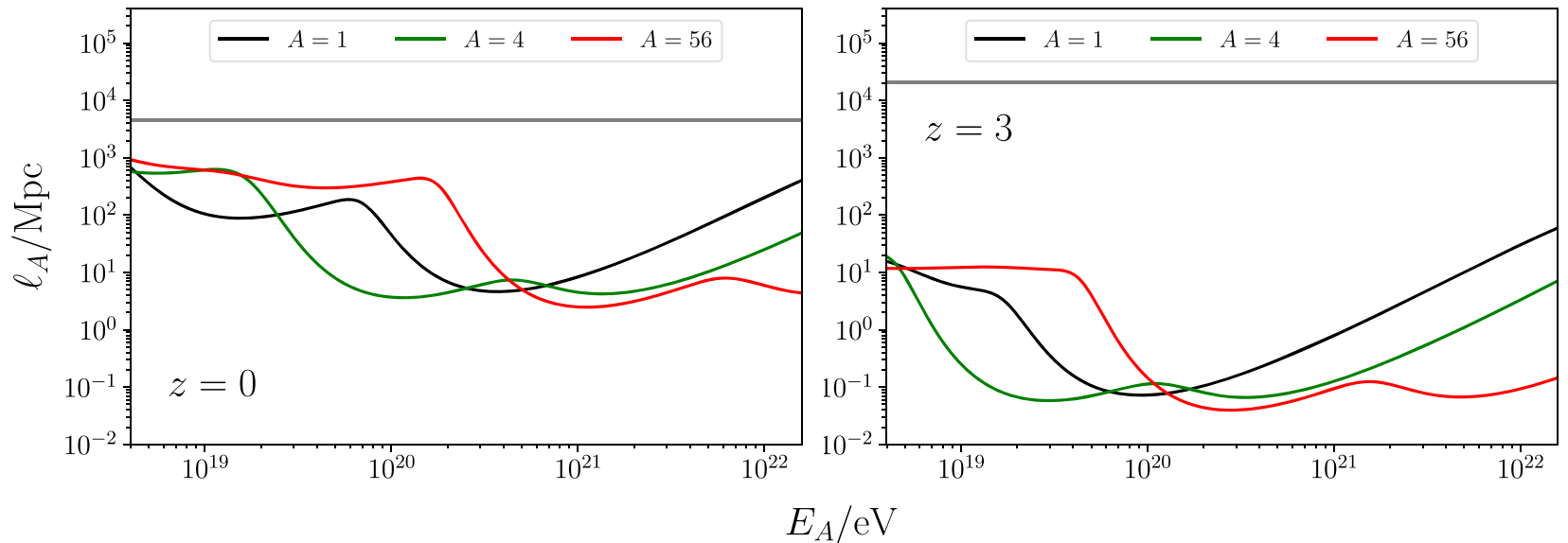

Figure 2. Total characteristic energy-loss distances of CR protons ( $\left({ }^{1} \mathrm{H}\right.$, black curves), helium $\left({ }^{4} \mathrm{He}\right.$, green curves), and iron $\left({ }^{56} \mathrm{Fe}\right.$, red curves) in background (CMB and EBL) cosmological radiation fields at $z=0$ (left panel) and $z=3$ (right panel). The corresponding energy-loss distances for adiabatic cooling due to cosmological expansion (gray lines) are shown for comparison. The cooling losses are stronger at $z=3$, because of a strong CMB radiation field, but the adiabatic losses are less severe.

pion production, or as $b^{\mathrm{sp}}{ }_{A} \approx-\epsilon_{A} \Lambda^{\mathrm{sp}}{ }_{A}$ for photospallation. ${ }^{18}$ Although approximated as continuous losses for the comparative purpose of Figures 1 and 2, our later calculations fully account for photopion and photospallation processes as absorption.

\section{Source Populations}

We adopt a parameterized model to describe the injection rate of CRs:

$$
\begin{aligned}
\mathcal{Q}^{\mathrm{a}}{ }_{A}\left(z, \epsilon_{A}, A\right) & =Q^{\mathrm{a}}{ }_{A}(1+z)^{-3} \\
& =\mathcal{C}_{0}(1+z)^{-3} \psi_{\mathrm{x}}(z) \frac{d}{d \epsilon_{A}}\left(\frac{d n^{\star}}{d t}\right),
\end{aligned}
$$

where $\mathcal{Q}^{\mathrm{a}}{ }_{A}$ and $Q^{\mathrm{a}}{ }_{A}$ are the co-moving and physical source terms, respectively. The normalization $\mathcal{C}_{0}$ is discussed in Section 3.2. The expression $\psi_{\mathrm{x}}(z)$ is the evolutionary function describing the distribution of a CR source population (the specific model is denoted by $\mathrm{x}$ ) with respect to redshift $z$ and is discussed in Section 3.1, and $d n^{\star} / d t$ is the volumetric injection rate of CRs by a source in that population.

\subsection{Redshift Distribution}

Four source populations are considered, and defined to a maximum redshift $z_{\max }=3$. This maximum redshift is chosen because it would be extremely unlikely that UHE CRs could reach us from greater distances, given their suppression by photo-hadronic interactions with the $\mathrm{CMB}$ and radiation emitted from astrophysical objects during their propagation in intergalactic space. Each of the source population models we consider follows a different redshift evolution. The first model (referred to as the star formation rate, or SFR, model) is based

\footnotetext{
18 This is only suitable as an approximation, and is not strictly valid as the fractional energy loss in a particular interaction could be substantial (see, e.g., Owen et al. 2018). Thus, particle energy losses are stochastic in nature, which introduces an additional statistical broadening to the absorption locations of UHE CRs impacted by this process. This broadening can change the mean path length by a factor of a few, and is particularly severe at high energies where the analytical path-length expression derived from the continuous approximation begins to differ substantially from that computed using a more appropriate numerical approach (Dermer \& Menon 2009).
}

on the redshift evolution of cosmic star formation (also adopted by Muzio et al. 2019), and takes the form:

$$
\psi_{\mathrm{SFR}}(z)=\psi_{\mathrm{SFR}}^{0} \frac{(1+z)^{k_{1}}}{1+\left[(1+z) / k_{2}\right]^{k_{3}}},
$$

where $k_{1}=2.7, k_{2}=2.9$, and $k_{3}=5.6$ are empirical fit parameters inherited from the best-fit function of Madau \& Dickinson (2014) (see also Robertson et al. 2015) ${ }^{19}$

Our second model choice is a parameterized redshift distribution of gamma-ray bursts (and is referred to as the GRB model hereafter). It follows the form presented in Wang et al. (2011):

$$
\psi_{\mathrm{GRB}}(z)=\psi_{\mathrm{GRB}}^{0}(1+z)^{k_{4}} \psi_{\mathrm{SFR}}(z),
$$

where $k_{4}=1.4$. This is based on indications from Swift observations that the GRB rate is enhanced relative to the SFR at high-redshift (Le \& Dermer 2007; see also Yüksel \& Kistler 2007) ${ }^{20}$

We introduce a third model to describe an AGN source population (referred to as the AGN source model hereafter). For this, we adopt the parameterization of Hasinger et al. (2005) (see also Ahlers et al. 2009; Wang et al. 2011; Alves Batista et al. 2019a),

$$
\psi_{\mathrm{AGN}}(z)=\psi_{\mathrm{AGN}}^{0} \begin{cases}(1+z)^{k_{5}} & \left(z<z_{1}\right) \\ z_{2}{ }^{k_{5}} & \left(z_{1} \leqslant z<z_{2}\right) \\ z_{2}{ }^{k_{5}} \cdot z_{2} z_{2}-z & \left(z \geqslant z_{2}\right)\end{cases}
$$

where $k_{5}=5.0, z_{1}=1.7, z_{2}=2.7$, which is largely consistent with the results in the later survey studies on AGN populations, e.g., Silverman et al. (2008), Ajello et al. (2012) (which were both adopted by Jacobsen et al. 2015, in the study of spectral and evolutionary properties of UHE neutrinos), and Ueda et al. (2014). There are various uncertainties in modeling other AGN

\footnotetext{
19 Alternative forms are adopted by some other researchers, for example, see Wang et al. (2011), Alves Batista et al. (2019a), and Palladino et al. (2020), which used the redshift distribution of Yüksel et al. (2008) instead.

${ }^{20}$ Note that alternative models are used in other studies to represent a GRB redshift distribution, for instance Alves Batista et al. (2019a) instead adopt the GRB source redshift distribution of Wanderman \& Piran (2010).
} 
Table 1

Summary of Parameter Choices Adopted for Each of the Four Redshift Source Distribution Models

\begin{tabular}{lcccc}
\hline \hline Model & Normalization $\psi_{\mathrm{x}}^{0}$ & $\begin{array}{c}\text { Spectral } \\
\text { index } \alpha\end{array}$ & $\log \left(R_{\mathrm{max}} / V\right)$ & $\begin{array}{c}\rho_{\mathrm{CR}} / 10^{48} \\
\mathrm{erg} \mathrm{Mpc}^{-3} \\
\mathrm{yr}^{-1}\end{array}$ \\
\hline (1) SFR & $\psi_{\mathrm{SFR}}^{0}=0.054$ & -1.3 & 18.2 & 0.5 \\
(2) GRB & $\psi_{\mathrm{GRB}}^{0}=0.013$ & -1.5 & 18.2 & 2.0 \\
(3) AGN & $\psi_{\mathrm{AGN}}^{0}=0.0041$ & -1.0 & 18.2 & 0.04 \\
(4) PLW & $\psi_{\mathrm{PLW}}^{0}=1.1$ & +1.0 & 18.7 & 15.0 \\
\hline
\end{tabular}

Note. See text for the choices of the model parameters.

contributions to the UHE CR flux. While it is unlikely that the results obtained from our parameterized model would need substantial revision, these uncertainties, e.g., those concerning the AGN evolution, could have detectable effects on the spectral properties of UHE CRs at Earth.

As the origins of UHE CRs are still to be determined, some studies adopted a simple power-law parameterization of the redshift evolution of the source model, in the form $(1+z)^{k_{\mathrm{PLW}}}$, where $k_{\mathrm{PLW}}$ is a fit parameter to be determined (e.g., Taylor et al. 2015; Aab et al. 2017; Jiang et al. 2021). Although our other three source models are more physically motivated, for comparison, we also consider such a power-law parameterization (referred to as the PLW model), defined as

$$
\psi_{\mathrm{PLW}}(z)=\psi_{\mathrm{PLW}}^{0}(1+z)^{k_{\mathrm{PLW}}},
$$

with $k_{\mathrm{PLW}}=-1.6$, the best-fit value obtained by Alves Batista et al. (2019a). In Equations (34)-(37), $\psi_{\mathrm{SFR}}^{0}, \psi_{\mathrm{GRB}}^{0}, \psi_{\mathrm{AGN}}^{0}$, and $\psi_{\mathrm{PLW}}^{0}$ are the normalization constants computed by integrating the respective source distribution over redshift up to a cutoff $\left(z_{\max }=3\right)$. Their resulting values are presented in Table 1 , and the redshift evolutionary histories for our four source models are shown in Figure 3.

\subsection{Cosmic-Ray Luminosity Density}

The normalization term $\mathcal{C}_{0}$ in Equation (33) can be determined from the UHE CR luminosity density, $\rho_{\mathrm{CR}}$. This is the rate of UHE CR energy density generated by the source population and, at $z=0$, it may be expressed as

$$
\rho_{\mathrm{CR}}=\left.\mathcal{C}_{0} \sum_{A} \int_{\epsilon_{\min }}^{\epsilon_{\max }} d \epsilon_{A} \epsilon_{A} \psi_{\mathrm{x}}(z) \frac{d}{d \epsilon_{A}}\left(\frac{d n^{\star}}{d t}\right)\right|_{z=0}
$$

(see Jiang et al. 2021). The exact value of $\rho_{\mathrm{CR}}$ is poorly constrained, however it may be roughly estimated by dividing the measured UHE CR energy density at $z=0$, around $10^{54} \mathrm{erg} \mathrm{Mpc}^{-3} \mathrm{dex}^{-1}$ (see Aab et al. 2020), by a typical CR energy-loss time, $\ell / c \sim 1 \mathrm{Gpc} / c \sim 1 \mathrm{Gyr}$ (see Figure 1), giving $\sim 10^{45} \mathrm{erg} \mathrm{Mpc}^{-3} \mathrm{yr}^{-1}$. This is comparable with more rigorous calculations (e.g., Berezinsky et al. 2006; Aloisio et al. 2007; Wang et al. 2011; Jiang et al. 2021), although the exact number is dependent on assumed model parameters, in particular, the lower energy cutoff of the CR spectrum, and the redshift distribution of the sources (Wang et al. 2011; Jiang et al. 2021). This variation is evident, as shown in the studies of Berezinsky et al. (2006) and Aloisio et al. (2007), where substantially larger values than our estimate, of $3.5 \times 10^{46} \mathrm{erg} \mathrm{Mpc}^{-3} \mathrm{yr}^{-1}$ and

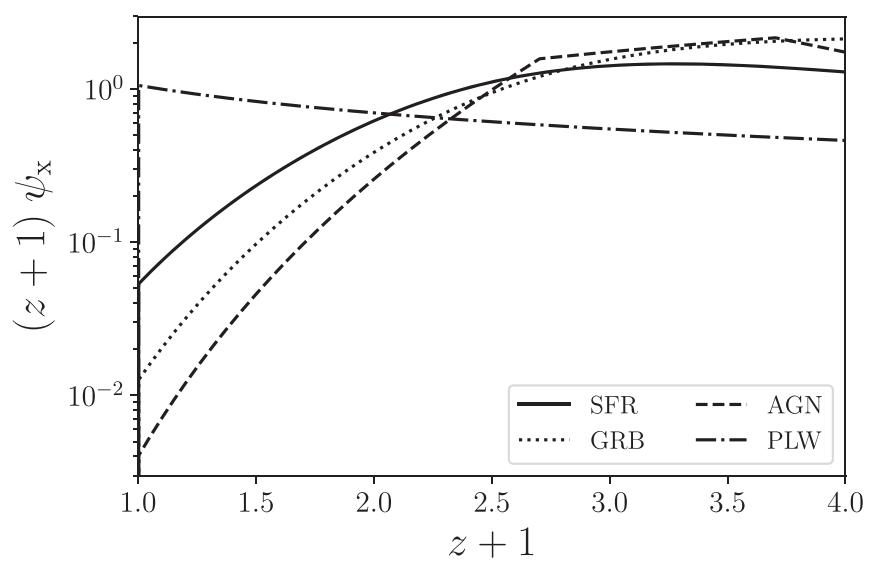

Figure 3. Redshift distributions of the four source models, SFR, GRB, AGN, and PLW. Their corresponding normalized redshift evolution functions are given in Equations (34), (35), (36), and (37), respectively.

$7 \times 10^{46} \mathrm{erg} \mathrm{Mpc}^{-3} \mathrm{yr}^{-1}$ (at energies above $10^{18} \mathrm{eV}$ ), respectively, are derived by assuming that there is no redshift dependence in the UHE CR source populations. By contrast, much lower values, in the range $0.2-2 \times 10^{44} \mathrm{erg} \mathrm{Mpc}^{-3} \mathrm{yr}^{-1}$ (at $3.16 \times 10^{19} \mathrm{eV}$ ), were obtained by Jiang et al. (2021), who adopted a negative redshift evolution for the UHE CR sources population. In Wang et al. (2011), a range of values depending on the assumed source model and energy cutoff are presented: for energies above $10^{18} \mathrm{eV}$, they found the source luminosity density to be $\sim 0.3-1 \times 10^{45} \mathrm{erg} \mathrm{Mpc}^{-3} \mathrm{yr}^{-1}$, for a source redshift distribution following that in Yüksel et al. (2008) (corresponding to the SFR scenario in this work); $\sim 6 \times 10^{44} \mathrm{erg} \mathrm{Mpc}^{-3} \mathrm{yr}^{-1}$ assuming a redshift distribution following the AGN scenario as in this work, cf Equation (36); and $\sim 0.8-3 \times 10^{45} \mathrm{erg} \mathrm{Mpc}^{-3} \mathrm{yr}^{-1}$ assuming a source distribution following that of Yüksel \& Kistler (2007) (cf. the GRB source scenario considered here). While we do not perform detailed model fitting, we set the UHE CR luminosity density for each of the source models to give a reasonable flux at $z=0$ when compared to Pierre Auger Observatory data (Verzi 2019); see Figures 5 and 6. The adopted values (at energies above $5 \times 10^{18} \mathrm{eV}$ ) are shown in Table 1 for each of the source models, and these were used to set the normalization $\mathcal{C}_{0}$ in Equation (38) above.

\subsection{Source Spectrum}

We model the energy spectrum of the injected CRs using the spectral form:

$$
\frac{d n^{\star}}{d \epsilon_{A}} \propto f_{A}\left(\frac{\epsilon_{A}}{\epsilon_{\min }}\right)^{-\alpha} \begin{cases}1 & (x<1) \\ \exp (1-x) & (x \geqslant 1)\end{cases}
$$

(Taylor et al. 2015; Alves Batista et al. 2019a; Jiang et al. 2021), where $x=\epsilon_{\mathrm{A}} m_{\mathrm{e}} c^{2} / Z_{\mathrm{A}} R_{\max }, R_{\max }(V)$ is the rigidity and the energy limits $\epsilon_{\min } m_{\mathrm{e}} c^{2}$ and $\epsilon_{\max } m_{\mathrm{e}} c^{2}$ retain their earlier values. Our parameter choices for the spectral index $\alpha$ and rigidity are shown in Table 1. Our adopted values are comparable to those derived from fits to Pierre Auger Observatory data in the analysis of Alves Batista et al. (2019a). We note that $\alpha$ is strongly dependent on the adopted source population's redshift evolution (see Taylor et al. 2015), leading to substantial differences in our choice of parameter value between the four models. 

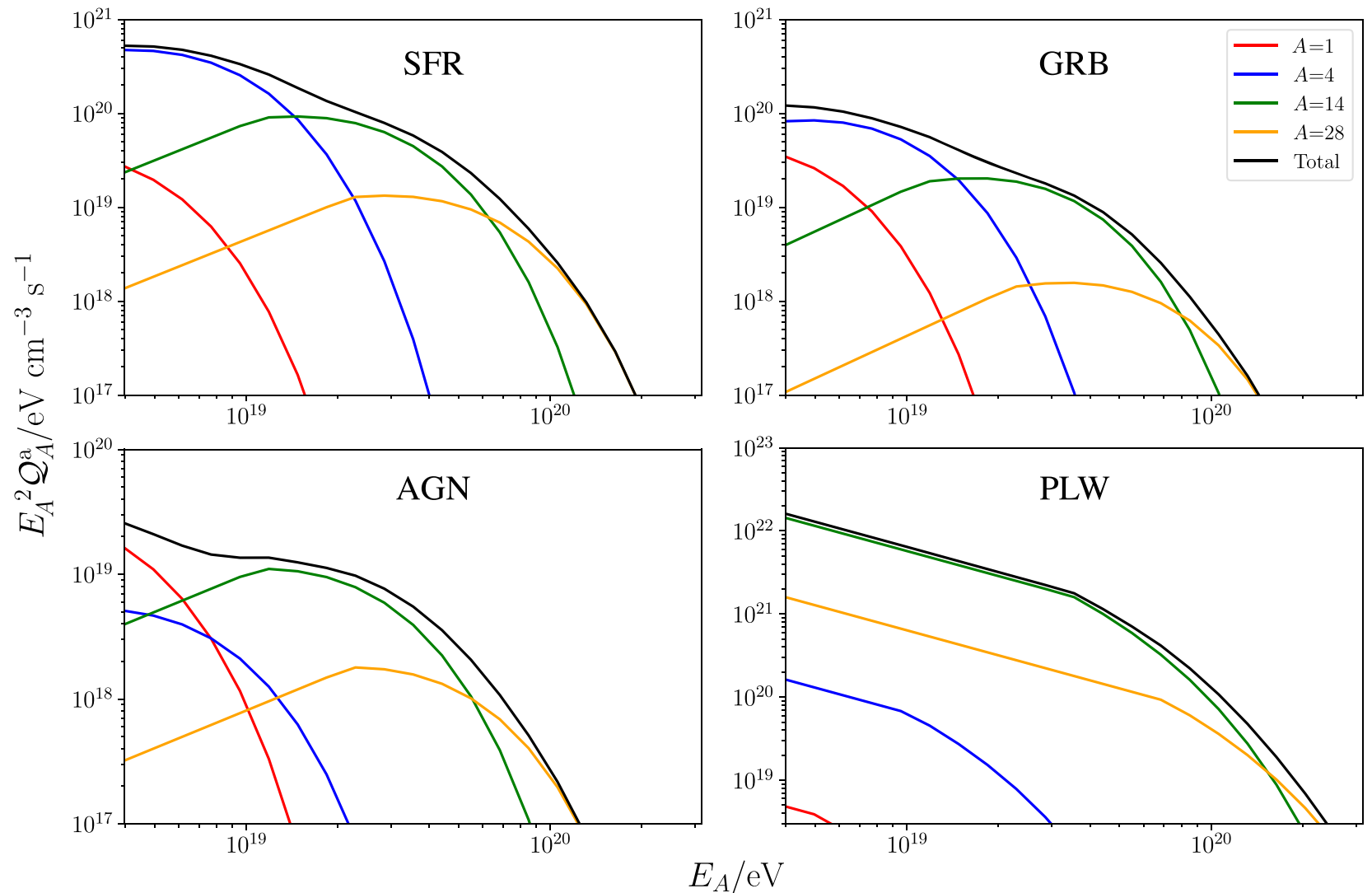

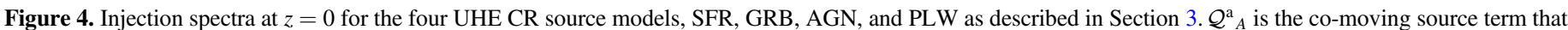

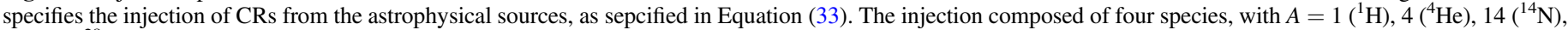
and $28\left({ }^{28} \mathrm{Si}\right)$, are shown. Their abundances are listed in Table 2 (which are the best-fit values from Alves Batista et al. 2019a).

\subsection{Injected Composition}

We adopt a simplified CR composition source model, where the full range of injected species are represented by the abundances of ${ }^{1} \mathrm{H},{ }^{4} \mathrm{He},{ }^{14} \mathrm{~N},{ }^{28} \mathrm{Si}$, and ${ }^{56} \mathrm{Fe}$. The individual component spectral forms are indicated in Figure 4, and their total contribution is normalized such that $f_{1}+f_{4}+f_{14}+f_{28}+f_{56}=1$. The fitted composition fraction values obtained by Alves Batista et al. (2019a) are adopted for species abundances for each of the four source populations (SFR, GRB, AGN, and PLW). The CR spectral models used in Alves Batista et al. (2019a) and this work are slightly different, but the fitted values given in Alves Batista et al. (2019a) are still a reasonable choice for our model. Note that we calculate the production of all secondary nuclear CR species between ${ }^{1} \mathrm{H}$ and ${ }^{56} \mathrm{Fe}$ formed as the primary UHE CRs propagate and interact with cosmological radiation fields. The abundance fractions of the species used in our calculations are shown in Table 2.

\section{Results and Discussion}

\subsection{Cosmic-Ray Spectrum and Composition}

The CR spectrum, expected to be observed at $z=0$, was computed by solving the transport equation (Equation (13) in Section 2.2) numerically using a Runge-Kutta integrator. ${ }^{21}$ Cases for the four source populations, SFR, GRB, AGN, and PLW, were calculated, with the numerical integration

\footnotetext{
21 An adaptive Runge-Kutta Fehlberg 4th order scheme (Press et al. 1992) was found to be sufficient.
}

Table 2

Abundance Fractions Adopted in the Model for the Four Different UHE CR Source Redshift Distributions

\begin{tabular}{lccccc}
\hline \hline$\psi_{\mathrm{x}}(z)$ model & $f_{1}\left({ }^{1} \mathrm{H}\right)$ & $f_{4}\left({ }^{4} \mathrm{He}\right)$ & $f_{14}\left({ }^{14} \mathrm{~N}\right)$ & $f_{28}\left({ }^{28} \mathrm{Si}\right)$ & $f_{56}\left({ }^{56} \mathrm{Fe}\right)$ \\
\hline (1) SFR & 0.1628 & 0.8046 & 0.0309 & 0.0018 & 0.0 \\
(2) GRB & 0.5876 & 0.3973 & 0.0147 & 0.0004 & 0.0 \\
(3) AGN & 0.8716 & 0.0778 & 0.0469 & 0.0038 & 0.0 \\
(4) PLW & 0.0003 & 0.0101 & 0.8906 & 0.0990 & 0.0 \\
\hline
\end{tabular}

proceeding from $z_{\max }=3$ to $z_{\min }=0$, with an explicit treatment of the injection of the CR compositions and of the evolution of the source populations along $z$. The computed CR flux and composition at $z=0$ took account of CR propagation and interaction effects, including cooling, absorption, and disintegration/spallation (outlined in Section 2). The results are are presented in Figure 5.

The production of secondary nuclei by photospallation when CRs propagate in intergalactic space drives the evolution of the composition of CRs, with heavy nuclei gradually being eroded to spawn lighter nuclear species, i.e., those of smaller atomic numbers. When the CRs are injected in our model, they are comprised of five nuclear species (i.e. ${ }^{1} \mathrm{H},{ }^{4} \mathrm{He},{ }^{14} \mathrm{~N},{ }^{28} \mathrm{Si}$, and ${ }^{56} \mathrm{Fe}$ ) as specified by the source models (see Section 3.4). Other species are produced via spallation as the CRs propagate from their sources to $z=0$. This evolution is captured in Figure 5, which shows the compositions in four broad bands to represent the binned distribution of nuclear species when the CRs complete their journey to reach $z=0$. 

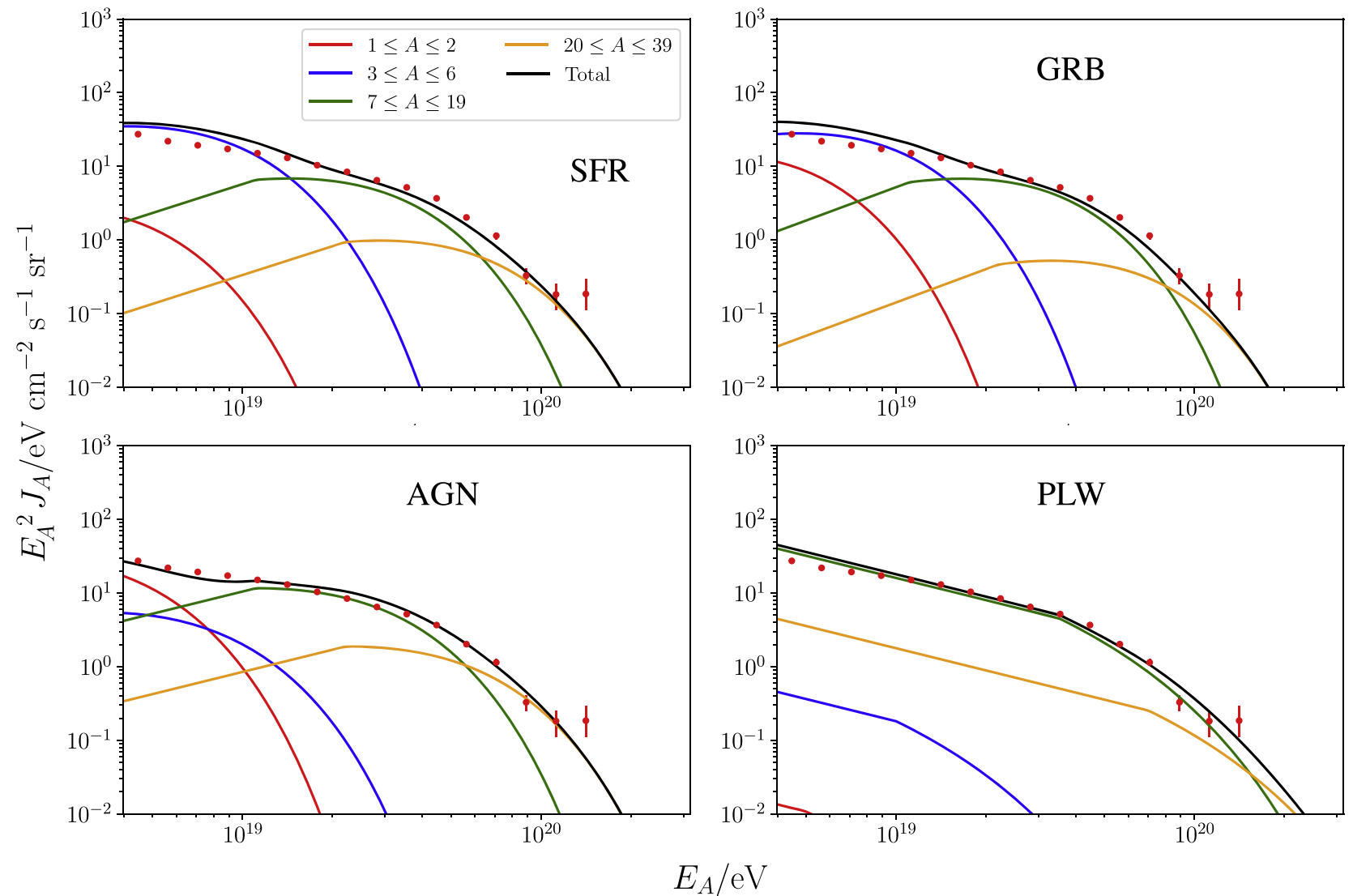

Figure 5. Flux spectra of CRs for the four source models, SFR, GRB, AGN, and PLW, propagated to $z=0$. The flux spectrum shown for the assigned range of $A$ has accounted for the production of secondary particles along the CR propagation. The data (discrete red data points with error bars) obtained by the Pierre Auger Observatory (Verzi 2019) are shown for comparison. The heaviest species of nucleus injected by the sources considered here is ${ }^{28} \mathrm{Si}$ in all the four models, i.e., all the nuclei have mass number $\mathrm{A} \leqslant 28$. The contribution from particles with $40 \leqslant A \leqslant 56$ is therefore zero.

When setting the abundance of ${ }^{56} \mathrm{Fe}$ to be zero, the heaviest species injected by the source model (see Section 3) is ${ }^{28} \mathrm{Si}$ $(A=28)$, so no CR nuclei would have $A>28$. As the CRs propagate, nuclei initially having $A=28$ would become nuclei with $A \leqslant 28$. The four broadband composition spectra in Figure 5 and the corresponding four injection species spectra in Figure 4 have strikingly similar morphology and relative strengths. The flux in the band $1 \leqslant A \leqslant 2$ is marginally higher, relatively, than the injected $\mathrm{H}$ fraction by the source model, while that in the $3 \leqslant A \leqslant 6,7 \leqslant A \leqslant 19$, and $20 \leqslant A \leqslant 39$ is marginally lower than the injected fractions of ${ }^{4} \mathrm{He},{ }^{14} \mathrm{~N}$, and ${ }^{28} \mathrm{Si}$, respectively. At $z=0$, the flux in each band is dominated by the injected source species, indicating that the evolution of species in an ensemble of CR nuclei propagating over vast distances through intergalactic space is only marginal (subject to the effects of cosmological expansion, cooling, absorption, and photospallation). We may therefore conclude that, in the absence of other additional factors, CR species arriving at $z=0$ are overwhelmingly primary CRs rather than secondaries reprocessed by photospallation. This result is not particularly surprising given that the injection process persists to $z=0$, and the rate of photospallation interactions is not comparable to the injection of primary CRs by the sources for the four populations considered. It also explains the limited evolution seen between the injected source spectrum and the post-propagation spectrum, with cooling rates being easily overcome by new injections at all energies (see Figure 1, which also clarifies that absorption processes dominate over any cooling process for CRs with energy above $10^{20} \mathrm{eV}$ ).

Our calculations for all considered models yield similar spectra at $z=0$ to that obtained by observations, and this agreement is evident (see Figure 5) when comparing the computed total flux spectra with data obtained by the Pierre Auger Observatory (PAO) (Verzi 2019). Figure 6 shows a comparison between the total flux spectra obtained for the four models, SFR, GRB, AGN, and PLW. The AGN source population model was found to produce (summed) spectra best matching the observation, while the flux levels in all four model cases are similar to the PAO observations and other studies (e.g., Alves Batista et al. 2019a). We note that an improved fit cannot be achieved with the parameter range considered in our models. Model refinement to obtain a better fit with available data is left to future studies.

Figure 7 further compares the average UHE CR spectral mass composition, $\langle\ln A\rangle$, for the four source classes with data (Pierre Auger Collaboration 2013; Yushkov 2019). This shows a clearer distinction between the different source classes, and also clearly rules out the PLW model as parameterized with Equation (37). The AGN model is also disfavored in this comparison (particularly at around $E_{\mathrm{A}} \sim 10^{19} \mathrm{eV}$ ), however 


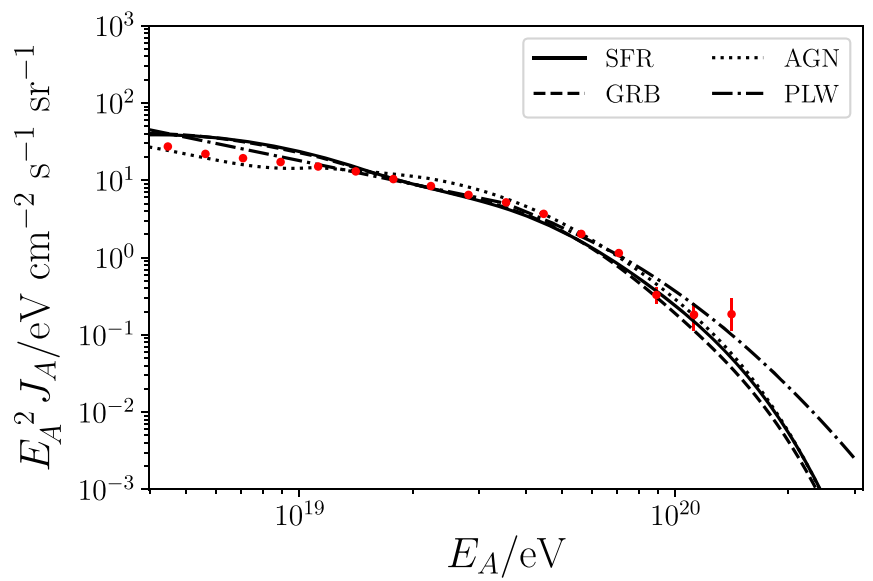

Figure 6. Flux spectra of CRs for the source models, SFR, GRB, AGN, and PLW. In each model, the flux is the total value obtained by summing the contributions of all the species considered in the calculations. The data (discrete red data points with error bars) obtained by the Pierre Auger Observatory (Verzi 2019) are shown for comparison.

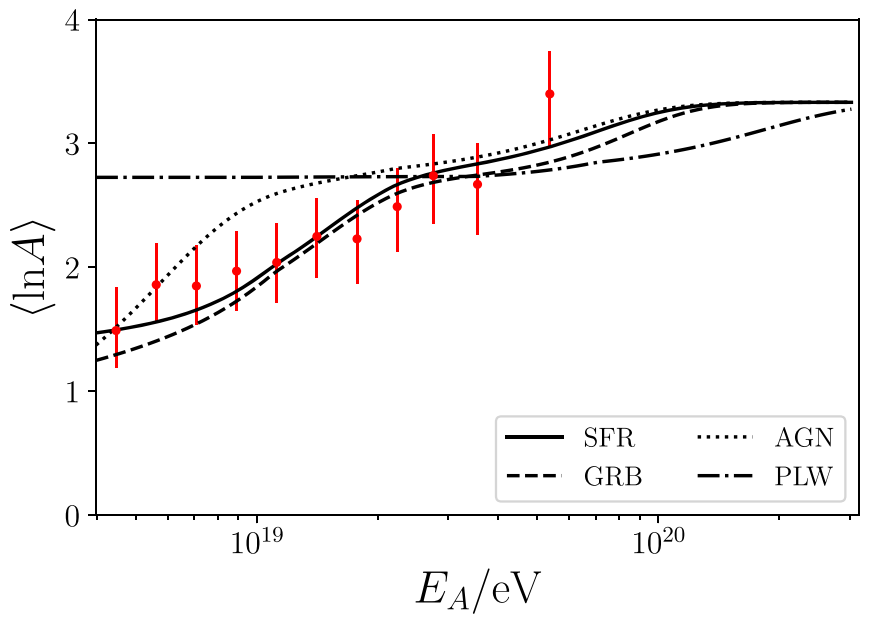

Figure 7. Average UHE CR mass composition, $\langle\ln A\rangle$, for the four source classes, compared to data obtained with the Pierra Auger Observatory (Pierre Auger Collaboration 2013; Yushkov 2019) when adopting the Sibyll 2.3c hadronic interaction model (Riehn et al. 2017).

possible variations in the adopted hadronic model, ${ }^{22}$ or the possibility of combined source classes with a refined AGN-type component may offer scope for an improved fit.

\subsection{Contributions from Distant UHE CR Sources}

Anisotropy in the arrival directions of UHE CRs above $8 \times 10^{18} \mathrm{eV}$, at a significance $>5.2 \sigma$ was recently reported (see Abreu et al. 2010; Aab et al. 2014, 2015, 2018; Pierre Auger Collaboration et al. 2017; Abbasi et al. 2018). There are also indications of correlations with certain candidate high-energy cosmic accelerators (see Pierre Auger Collaboration et al. 2007; Abreu et al. 2010), which include AGN detected in $\gamma$-rays and X-rays (Nemmen et al. 2010; Terrano et al. 2012), ${ }^{23}$ as well as source distributions tracing large-scale structures, with a source

\footnotetext{
${ }^{22}$ The data shown are computed using the Sibyll $2.3 \mathrm{c}$ hadronic interaction model (Riehn et al. 2017), however other models are available (e.g., Ostapchenko 2011; Pierog et al. 2015), and these yield slightly different $\langle\ln A\rangle$ spectral shapes and normalizations.

23 Note that this is in contrast with the finding of Mirabal \& Oya (2010) (see also Álvarez et al. 2016).
}

population being biased with respect to the distribution of galaxies (Kashti \& Waxman 2008). A CR "hotspot" was recently reported by Abbasi et al. (2014). Studies by Fang et al. (2014) and $\mathrm{He}$ et al. (2016) showed that this hotspot is consistent with a single source rather than a chance signal. Clusters of excess arrivals of UHE CRs above an isotropic background from the direction of Centaurus $A^{24}$ have been found in some analyses (Fargion \& D'Armiento 2011; Kim 2013a, 2013b). ${ }^{25}$ However, there is no clear detection of excesses around other nearby radio galaxies, e.g., Centaurus B and Virgo A (see Fraija et al. 2019; Kobzar et al. 2019).

If UHE CRs are produced by sources located nearby to our Galaxy with a fraction originating from within the conventional GZK horizon, as well as sources at large (cosmological) distances, then their arrival flux could have two components: an isotropic component corresponding to unresolved distant sources and an anisotropic component associated with relatively nearby sources. The reported anisotropies detected in UHE CRs are consistent with this scenario, and consideration of a foreground and background component in the UHE CR flux was shown in Kim \& Kim (2011) to improve anisotropy correlations with the positions of potential nearby sources. This is complimented by the blind test performed by Rubtsov et al. (2012) for verifying the hypothesis that AGN were the origin of UHE CRs, using event sets from Yakutsk, AGASA, and HiRes. Their findings were consistent with a random background (see also High Resolution Fly'S Eye Collaboration et al. 2008, which also found 13 UHE CR events detected by HiRes in the northern hemisphere to be consistent with isotropy).

Here, we assess the fraction of UHE CR flux that could originate at large distances, from sources located beyond a GZK "horizon" distance of a few tens of megaparsecs (see, e.g., Kachelrieß \& Semikoz 2019), and determine their contribution to a UHE CR background. Distant CR sources are homogeneously distributed across the celestial sphere over a range of redshifts. Regardless of the fraction of their flux that can survive to reach Earth, CRs originating from these sources should not, at least in principle, show resolvable anisotropy. To assess the relative contributions to the CRs observed at $z=0$ from the CRs initially produced by distant sources at different redshifts, we computed the flux spectra (for each of the source models) with several assigned $z_{\max }$, taking values from 3.0, 2.0, 1.0 , and 0.5 . The spectra computed at $z=0$ for four source models are presented in Figure 8 for four values of $z_{\max }$. This shows that changing either the source model or $z_{\max }$ leads to discernible changes in the spectra at $z=0$. The contribution of distant source populations is clearly substantial and naturally accounts for the emergence of an isotropic (background) component in the observed UHE CR flux on Earth.

The significance of the distant sources' contribution varies among the different source evolution models, which can be seen from comparing the set of spectra of different $z_{\max }$ shown in different panels of Figure 8. The amount of reduction between the spectrum from sources below $z=3$ to that from sources below $z=2$ accounts for a large fraction of the total CR flux arriving at $z=0$, which is at a level between $3 \%$ and

\footnotetext{
${ }^{24}$ Centaurus A has been considered as a source able to accelerate CRs to energies of $\sim 10^{20} \mathrm{eV}$ at distances above $100 \mathrm{kpc}$ from its core (Pe'er \& Loeb 2012).

${ }^{25}$ Accounting also for a smearing effect due to intergalactic magnetic fields of order $10 \mathrm{nG}$ (Kim 2013b).
} 

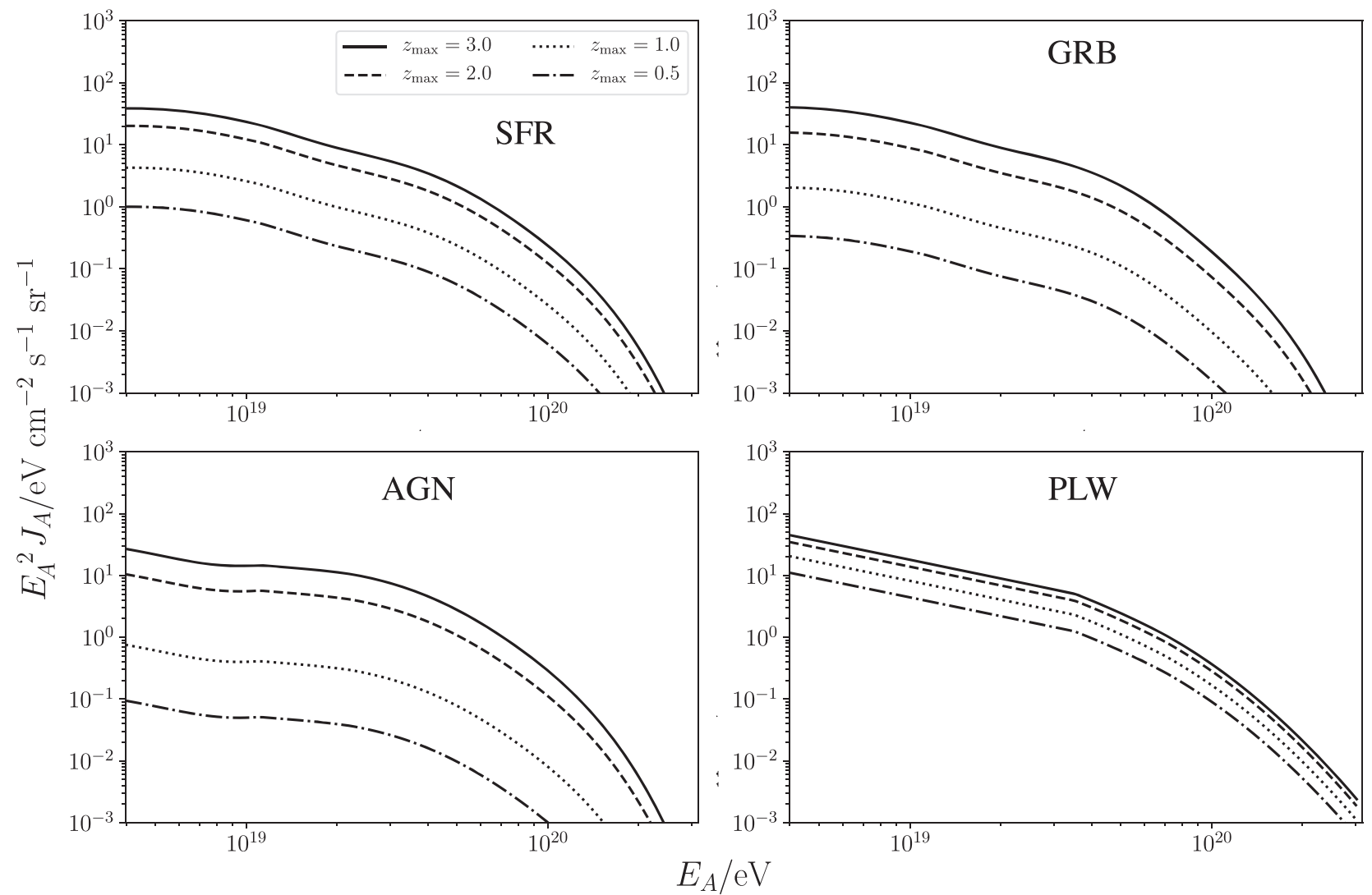

Figure 8. Total flux spectra of CRs propagated to $z=0$ for the four source models. In each model, the cases for CR injection truncated at redshifts $z_{\max }=0.5,1.0,2.0$, and 3.0 (curves from bottom to top) are shown. Comparing the flux spectra for different redshift truncation reveals that the contributions from distance sources are significant.

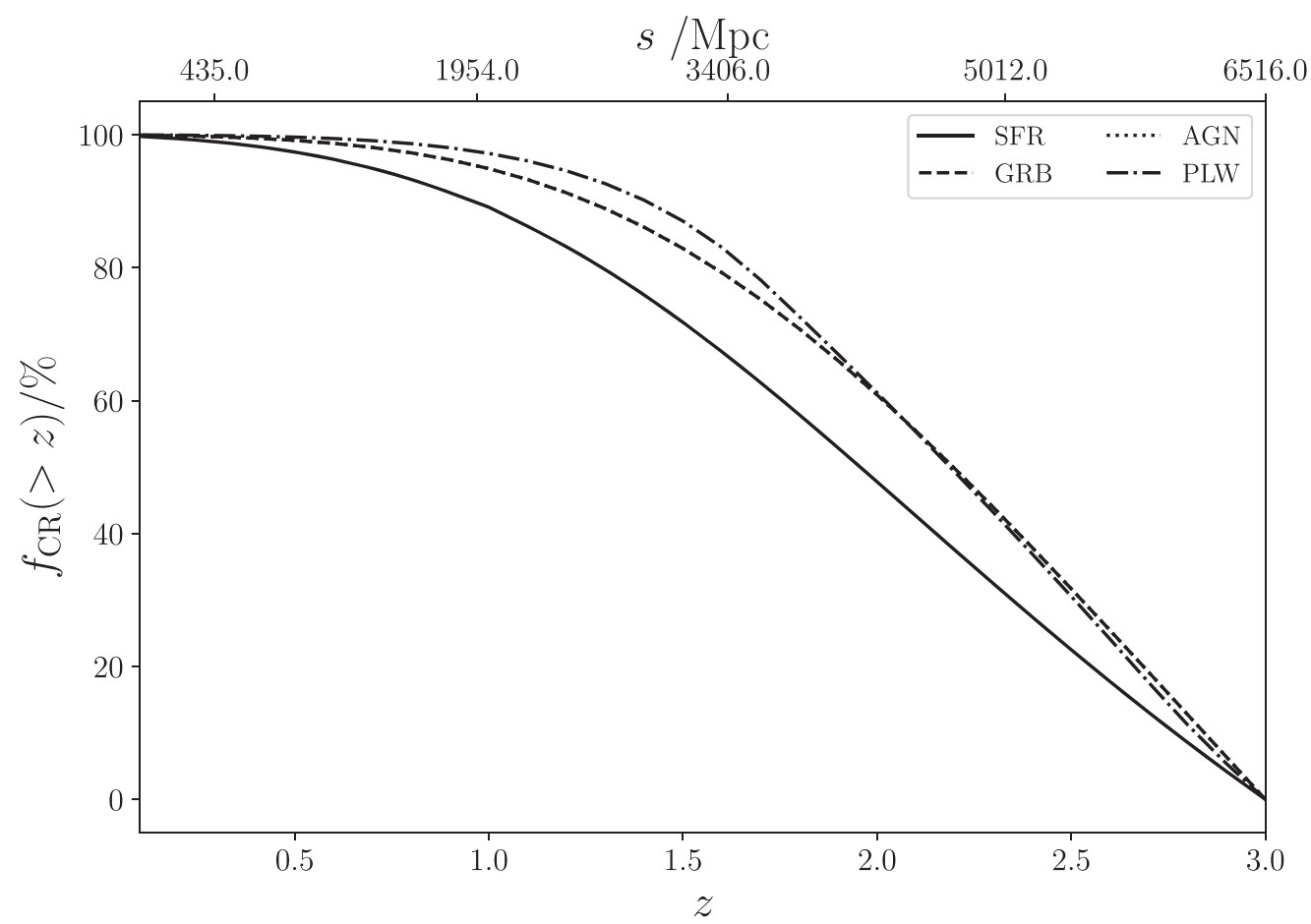

Figure 9. The fraction of CRs that originate from redshift higher than $z, f_{\mathrm{CR}}(>z)$, for the four source models. The bottom abscissa shows the redshift $z$, and the top abscissa the corresponding co-moving distances, for the cosmological model with $\Omega_{\mathrm{m}, 0}=0.315 \pm 0.007, \Omega_{\mathrm{r}, 0} \approx 0, \Omega_{\Lambda, 0}=0.685 \pm 0.007$, and $h=0.673 \pm 0.006$ (see Section 2.2). The curves for the GRB and AGN models are almost indistinguishable, a consequence of the redshift evolution of GRB and AGN, in that most contributions from their respective sources are located at similar redshifts (see Figure 3). 
$50 \%$ in the SFR, GRB, and AGB models. The PLW model however, has a relatively smaller reduction in the flux for the same redshift interval, implying that $\mathrm{CR}$ flux is dominated by sources at lower redshifts in that case.

Figure 9 shows the fractional contribution from sources above a given redshift, $f_{\mathrm{CR}}(>z)$, for the four source models. Most noticeably, the curves for the GRB and AGN models are practically indistinguishable. The morphology of the $f_{\mathrm{CR}}(>z)$ curve is a manifestation of the redshift locations where a specific population of sources has the most significant contribution. The dominant redshift locations of the GRB and AGN CR sources are very similar to one another (cf. the curves of these two source populations in Figure 3, particularly above $(z+1) \approx 2.5)$. This, together with the lacking of substantial numbers of GRB and AGN below $(z+1) \sim 2.5$ leads to their almost identical $f_{\mathrm{CR}}(>z)$ curves. Figure 9 indicates that, for all the source population models considered, a large faction of UHE CR flux observed at Earth would originate from regions far beyond the conventional GZK horizon of a few tens of megaparsecs, and would not be attributed to local sources. If the UHE CRs are predominantly produced as a consequence of star formation, which is represented by the SFR model here, then $\sim 50 \%$ of the CRs could originate from as far as $z \sim 2$ or above.

These findings are robust. The results obtained from our calculations are not sensitive to the assumed composition of the injected UHE CR flux. The outcome is similar even if the CR injections are restricted to a single species (of any of the five nuclei considered). This is in line with the chemical evolution due to photospallation processes being insignificant, otherwise, the composition evolution of CRs would be strongly manifested in the CR energy spectra of the species observed at Earth.

\section{Summary and Conclusions}

In this work, we investigated the UHE CR flux and spectra at $z=0$ with the explicit considerations of (i) the distribution of candidate sources in the cosmological context (modeled as four different redshift-dependent populations, denoted as SFR, GRB, AGN, and PLW as described in previous sections), (ii) the composition of the injected CR nuclear species (specified by the abundance of ${ }^{1} \mathrm{H},{ }^{4} \mathrm{He},{ }^{28} \mathrm{Si}$, and ${ }^{56} \mathrm{Fe}$ species), and (iii) the relevant radiative and cooling processes and particle absorption interactions (which include photospallation for the CR nuclei when interacting with $\mathrm{CMB}$ and $\mathrm{EBL}$ radiation fields). We solved the particle-transport equations numerically and determined the evolution of CR properties, in particular the CR flux and spectra and the compositions of species for each model. Our calculations have shown that sources at redshifts as high as $z \sim(2-3)$ can contribute substantially to UHE CRs detected on Earth, with different source classes being distinguishable using the average UHE CR mass composition spectrum at $z=0$. Comparison with average $\mathrm{CR}$ mass data obtained from the Pierre Auger Observatory allowed a strong contribution from a PLW-type source population (as parameterized with Equation 37) to be ruled out.

Regardless of population class, we find that most of the UHE CRs from these distant source populations are primary particles, despite the large cosmological distances that they have traversed. These UHE CRs are diffuse and isotropic, as they are of cosmological origin. They constitute the isotropic background on which an anisotropic UHE CR component associated with the nearby $\mathrm{CR}$ accelerators is superimposed.

E.R.O. is supported by the Center for Informatics and Computation in Astronomy (CICA) at National Tsing Hua University (NTHU), funded by the Ministry of Education of Taiwan (ROC). Y.X.J.Y. is supported by a NTHU International Student Scholarship and by a grant from the Ministry of Science and Technology of Taiwan (ROC), 109-2628-M-007005-RSP (PI Prof. Albert Kong). This work used highperformance computing facilities operated by CICA at NTHU. This equipment was funded by the Ministry of Education of Taiwan and the Ministry of Science and Technology of Taiwan. The authors thank the anonymous referee for their comments, which substantially improved the manuscript. This research has made use of NASA's Astrophysics Data Systems.

\section{Appendix \\ Extragalactic Background Light}

Extragalactic background light is composed of radiation emitted from astrophysical objects. Its energy is mainly concentrated in two spectral peaks: one at optical wavelengths, being broadly associated with stellar emission from within populations of galaxies, while the other is at infrared wavelengths, and is presumably dominated by dust-reprocessed astrophysical emission (also mainly originating from within galaxies).

The EBL is well studied observationally in the local Universe (see Cooray 2016). However, its redshift evolution is less well constrained (for EBL constraints from observations over a range of redshifts, see, e.g., Franceschini \& Rodighiero 2017), and is determined by the evolution of its astrophysical source populations. While many approaches have been taken to model the cosmological evolution of the EBL in detail, including forward-evolutionary models (e.g., Finke et al. 2010; Kneiske \& Dole 2010), backward-evolutionary models (e.g., Domínguez et al. 2011; Stecker et al. 2012), and semi-analytical models (e.g., Gilmore et al. 2012; Inoue et al. 2013), these are each subject to certain inherent assumptions and uncertainties leading to substantial variations in predictions between models and modeling approaches. Although the EBL has been shown to affect the cosmological propagation of UHE CRs (e.g., Allard et al. 2005; Aloisio et al. 2013b), and the exact form of model adopted has been demonstrated to have nonnegligible effects on the spectrum of UHE CRs (e.g., Aloisio et al. 2013b), we do not find it to have substantial impacts on the results of this study, with any EBL model (if reasonable) yielding comparable results. We therefore adopt a simple analytic representation of the EBL, comprised of six superposed blackbody components, as listed in Table 3. Of these, one is attributed to dust (infrared) emission and the remaining five are a non-physical approximation to the UV/ optical part of the EBL that presumably arises from stellar emission. Of these five, only the components "UV/O (1)" and "UV/O (2)" fall within the photon energy range relevant to our 
Table 3

Summary of Parameter Choices for $z=0$ EBL Model, Approximating an Upper-estimate for the EBL Using Blackbody Components (Hauser \& Dwek 2001)

\begin{tabular}{lccc}
\hline \hline Component $^{\mathrm{a}}$ & $T / K$ & $\Theta_{i}$ & $f_{i, 0}$ \\
\hline Dust & 62 & $1.1 \times 10^{-8}$ & $3.5 \times 10^{-7}$ \\
UV/O (1) & 400 & $6.7 \times 10^{-8}$ & $9.0 \times 10^{-12}$ \\
UV/O (2) & 1,000 & $1.7 \times 10^{-7}$ & $4.5 \times 10^{-13}$ \\
UV/O (3) & 3,000 & $5.1 \times 10^{-7}$ & $1.4 \times 10^{-14}$ \\
UV/O (4) & 5,500 & $9.3 \times 10^{-7}$ & $6.0 \times 10^{-16}$ \\
UV/O (5) & 12,000 & $2.0 \times 10^{-6}$ & $6.1 \times 10^{-17}$ \\
\hline
\end{tabular}

Note. Dimensionless weights $f_{i, 0}$ have been estimated from relative normalizations.

${ }^{a}$ Note that the UV/O components are not physically motivated. These are adopted to provide a sufficient approximation to the EBL spectrum in the energy range of interest while affording analytical tractability.

calculations. ${ }^{26}$ The components $i$ each take the form

$$
n_{i}\left(\epsilon_{\mathrm{ph}}, z\right)=\frac{8 \pi f_{i}(z)}{\lambda_{\mathrm{c}}^{3}} \frac{\epsilon_{\mathrm{ph}}^{2}}{\exp \left(\epsilon_{\mathrm{ph}} / \Theta_{i}\right)-1},
$$

where terms retain their earlier definitions. The redshiftdependent dimensionless weights of the components are

$$
f_{i}(z)=f_{i, 0}\left\{\begin{array}{l}
(1+z)^{3.1}, \quad z<z_{\text {cut }} \\
\left(1+z_{\text {cut }}\right)^{3.1}, \quad z \geqslant z_{\text {cut }}
\end{array}\right.
$$

up to $z=3$, where $z_{\text {cut }}=1.4$, which follows the "baseline" EBL redshift evolution model in Aloisio et al. (2013b). More detailed investigation of the impact of the redshift evolution of the EBL on UHE CR propagation falls beyond the scope of this paper, and is more appropriate for a dedicated future study. Temperature, energy density, dimensionless temperature $\Theta_{i}$ and $z=0$ dimensionless weights $f_{i, 0}$ for each EBL model component $i$ are listed in Table 3 . These choices have been shown to give reasonable consistency with local observational constraints (Hauser \& Dwek 2001). Note that, for comparison, in the case of the CMB, $f=1$ and $\Theta(z)=4.58 \times 10^{-10}(1+z)$.

\section{ORCID iDs}

Ellis R. Owen (1) https://orcid.org/0000-0003-1052-6439

Qin Han (ㄴ) https://orcid.org/0000-0002-2342-9956

Kinwah Wu (i) https://orcid.org/0000-0002-7568-8765

Y. X. Jane Yap (10) https://orcid.org/0000-0002-3349-3089

Pooja Surajbali (i) https://orcid.org/0000-0002-8516-6469

\section{References}

Aab, A., Abreu, P., Aglietta, M., et al. 2014, ApJ, 794, 172 Aab, A., Abreu, P., Aglietta, M., et al. 2015, ApJ, 804, 15 Aab, A., Abreu, P., Aglietta, M., et al. 2017, JCAP, 2017, 038 Aab, A., Abreu, P., Aglietta, M., et al. 2018, ApJ, 868, 4

Aab, A., Abreu, P., Aglietta, M., et al. 2020, PhRvL, 125, 121106 Abbasi, R. U., Abe, M., Abu-Zayyad, T., et al. 2014, ApJL, 790, L21 Abbasi, R. U., Abe, M., Abu-Zayyad, T., et al. 2018, ApJ, 858, 76 Abreu, P., Aglietta, M., Ahn, E. J., et al. 2010, APh, 34, 314 Abu-Zayyad, T., Aida, R., Allen, M., et al. 2013, ApJL, 768, L1

\footnotetext{
${ }^{26}$ Our choice of multiple un-physical blackbody components for the UV/ optical region of the EBL is adopted for analytical tractability in this work; however, more physically representative modified blackbody approximations for the EBL have been adopted in other works (e.g., Dermer \& Menon 2009).
}

Ahlers, M., Anchordoqui, L. A., \& Sarkar, S. 2009, PhRvD, 79, 083009

Ahlers, M., \& Salvado, J. 2011, PhRvD, 84, 085019

Ajello, M., Shaw, M. S., Romani, R. W., et al. 2012, ApJ, 751, 108

Allard, D., Ave, M., Busca, N., et al. 2006, JCAP, 2006, 005

Allard, D., Parizot, E., Olinto, A. V., Khan, E., \& Goriely, S. 2005, A\&A, 443, L29

Aloisio, R., Berezinsky, V., \& Blasi, P. 2014, JCAP, 2014, 020

Aloisio, R., Berezinsky, V., Blasi, P., et al. 2007, APh, 27, 76

Aloisio, R., Berezinsky, V., \& Grigorieva, S. 2013a, APh, 41, 73

Aloisio, R., Berezinsky, V., \& Grigorieva, S. 2013b, APh, 41, 94

Álvarez, E., Cuoco, A., Mirabal, N., \& Zaharijas, G. 2016, JCAP, 2016, 023

Alves Batista, R., Biteau, J., Bustamante, M., et al. 2019b, FrASS, 6, 23

Alves Batista, R., de Almeida, R. M., Lago, B., \& Kotera, K. 2019a, JCAP, 2019, 002

Alves Batista, R., Erdmann, M., Evoli, C., et al. 2013, in Int. Cosmic Ray Conf., 33, 694

Berezinsky, V., Gazizov, A., \& Grigorieva, S. 2006, PhRvD, 74, 043005

Berezinsky, V. S., \& Gazizov, A. Z. 1993, PhRvD, 47, 4206

Berezinsky, V. S., \& Grigor'eva, S. I. 1988, A\&A, 199, 1

Bethe, H., \& Heitler, W. 1934, RSPSA, 146, 83

Bethe, H. A., \& Maximon, L. C. 1954, PhRv, 93, 768

Bird, D. J., Corbato, S. C., Dai, H. Y., et al. 1994, ApJ, 424, 491

Bird, D. J., Corbato, S. C., Dai, H. Y., et al. 1995, ApJ, 441, 144

Blumenthal, G. R. 1970, PhRvD, 1, 1596

Cooray, A. 2016, RSOS, 3, 150555

Deligny, O., Kawata, K., \& Tinyakov, P. 2017, PTEP, 2017, 12A104

Dermer, C. D., \& Menon, G. 2009, High energy radiation from black holes: gamma rays, cosmic rays, and neutrinos, Princeton series in astrophysics (Princeton, NJ: Princeton Univ. Press)

Domínguez, A., Primack, J. R., Rosario, D. J., et al. 2011, MNRAS, 410, 2556

Fang, K., Fujii, T., Linden, T., \& Olinto, A. V. 2014, ApJ, 794, 126

Fargion, D., \& D'Armiento, D. 2011, in Italian Physical Society Conf. Proc. 103

Finke, J. D., Razzaque, S., \& Dermer, C. D. 2010, ApJ, 712, 238

Fraija, N., Araya, M., Galván-Gámez, A., \& de Diego, J. A. 2019, JCAP, 2019, 023

Franceschini, A., \& Rodighiero, G. 2017, A\&A, 603, A34

Giacinti, G., Kachelrieß, M., Semikoz, D. V., \& Sigl, G. 2012, JCAP, 2012, 031

Gilmore, R. C., Somerville, R. S., Primack, J. R., \& Domínguez, A. 2012, MNRAS, 422, 3189

Gould, R. J. 1975, ApJ, 196, 689

Gould, R. J. 1993, ApJ, 417, 12

Greisen, K. 1966, PhRvL, 16, 748

Hasinger, G., Miyaji, T., \& Schmidt, M. 2005, A\&A, 441, 417

Hauser, M. G., \& Dwek, E. 2001, ARA\&A, 39, 249

Hayward, E. 1960, Sci, 132, 951

He, H.-N., Kusenko, A., Nagataki, S., et al. 2016, PhRvD, 93, 043011

High Resolution Fly'S Eye Collaboration, Abbasi, R. U., Abu-Zayyad, T., et al. 2008, APh, 30, 175

Hillas, A. M. 1984, ARA\&A, 22, 425

Hümmer, S., Rüger, M., Spanier, F., \& Winter, W. 2010, ApJ, 721, 630

Inoue, Y., Inoue, S., Kobayashi, M. A. R., et al. 2013, ApJ, 768, 197

Ivanov, D. 2017, PoS, ICRC2017, 498

Jacobsen, I. B., Wu, K., On, A. Y. L., \& Saxton, C. J. 2015, MNRAS, 451, 3649

Jiang, Y., Zhang, B. T., \& Murase, K. 2021, PhRvD, 104, 043017

Jost, R., Luttinger, J. M., \& Slotnick, M. 1950, PhRv, 80, 189

Kachelrieß, M., \& Semikoz, D. V. 2019, PrPNP, 109, 103710

Kalashev, O. E., Khrenov, B. A., Klimov, P., Sharakin, S., \& Troitsky, S. V. 2008, JCAP, 2008, 003

Kampert, K.-H., Kulbartz, J., Maccione, L., et al. 2013, APh, 42, 41

Karakula, S., \& Tkaczyk, W. 1993, APh, 1, 229

Kashti, T., \& Waxman, E. 2008, JCAP, 2008, 006

Kim, H. B. 2013a, JKPS, 62, 708

Kim, H. B. 2013b, ApJ, 764, 121

Kim, H. B., \& Kim, J. 2011, JCAP, 2011, 006

Klein, S. R. 2006, RaPC, 75, 696

Kneiske, T. M., \& Dole, H. 2010, A\&A, 515, A19

Kobzar, O., Hnatyk, B., Marchenko, V., \& Sushchov, O. 2019, MNRAS, 484,1790

Kotera, K., \& Olinto, A. V. 2011, ARA\&A, 49, 119

Le, T., \& Dermer, C. D. 2007, ApJ, 661, 394

Madau, P., \& Dickinson, M. 2014, ARA\&A, 52, 415

Mirabal, N., \& Oya, I. 2010, MNRAS, 405, L99 
Moncada, R. J., Colon, R. A., Guerra, J. J., O’Dowd, M. J., \& Anchordoqui, L. A. 2017, JHEAp, 13, 32

Morejon, L., Fedynitch, A., Boncioli, D., Biehl, D., \& Winter, W. 2019, JCAP, 2019, 007

Mücke, A., Rachen, J. P., Engel, R., Protheroe, R. J., \& Stanev, T. 1999, PASA, 16, 160

Muzio, M. S., Unger, M., \& Farrar, G. R. 2019, PhRvD, 100, 103008

Nakamura, K. 2010, JPhG, 37, 075021

Nemmen, R. S., Bonatto, C., \& Storchi-Bergmann, T. 2010, ApJ, 722, 281

Ostapchenko, S. 2011, PhRvD, 83, 014018

Owen, E. R., Jacobsen, I. B., Wu, K., \& Surajbali, P. 2018, MNRAS, 481, 666

Owen, E. R., Lee, K.-G., \& Kong, A. K. H. 2021, MNRAS, 506, 52

Owen, E. R., Wu, K., Jin, X., Surajbali, P., \& Kataoka, N. 2019, A\&A, 626, A85

Palladino, A., van Vliet, A., Winter, W., \& Franckowiak, A. 2020, MNRAS, 494, 4255

Peacock, J. A. 1999, Cosmological Physics (Cambridge: Cambridge Univ. Press)

Pe'er, A., \& Loeb, A. 2012, JCAP, 2012, 007

Pierog, T., Karpenko, I., Katzy, J. M., Yatsenko, E., \& Werner, K. 2015, PhRvC, 92, 034906

Pierre Auger Collaboration 2013, JCAP, 2013, 026

Pierre Auger Collaboration, Aab, A., Abreu, P., et al. 2017, Sci, 357, 1266

Pierre Auger Collaboration, Abraham, J., Abreu, P., et al. 2007, Sci, 318, 938

Planck Collaboration, Aghanim, N., Akrami, Y., et al. 2020, A\&A, 641, A6

Press, W. H., Teukolsky, S. A., Vetterling, W. T., \& Flannery, B. P. 1992, Numerical recipes in FORTRAN. The Art of Scientific Computing (Cambridge: Cambridge Univ. Press)

Protheroe, R. J., \& Johnson, P. A. 1996, APh, 4, 253

Puget, J. L., Stecker, F. W., \& Bredekamp, J. H. 1976, ApJ, 205, 638
Rieger, F. 2019, High Energy Phenomena in Relativistic Outflows VII (Trieste: SISSA), 19

Riehn, F., Dembinski, H., Fedynitch, A., et al. 2017, PoS, ICRC2017, 301

Robertson, B. E., Ellis, R. S., Furlanetto, S. R., \& Dunlop, J. S. 2015, ApJL, 802, L19

Rodrigues, X., Heinze, J., Palladino, A., van Vliet, A., \& Winter, W. 2021, PhRvL, 126, 191101

Romero, G., \& Gutiérrez, E. 2020, Univ, 6, 99

Rubtsov, G. I., Tkachev, I. I., \& Dolgov, A. D. 2012, JETPL, 95, 501

Silverman, J. D., Green, P. J., Barkhouse, W. A., et al. 2008, ApJ, 679, 118

Stecker, F. 1969, PhRv, 180, 1264

Stecker, F. W. 1979, ApJ, 228, 919

Stecker, F. W., Malkan, M. A., \& Scully, S. T. 2012, ApJ, 761, 128

Stecker, F. W., \& Salamon, M. H. 1999, ApJ, 512, 521

Stepney, S., \& Guilbert, P. W. 1983, MNRAS, 204, 1269

Taylor, A. M., Ahlers, M., \& Hooper, D. 2015, PhRvD, 92, 063011

Terrano, W. A., Zaw, I., \& Farrar, G. R. 2012, ApJ, 754, 142

Ueda, Y., Akiyama, M., Hasinger, G., Miyaji, T., \& Watson, M. G. 2014, ApJ, 786, 104

Unger, M., Farrar, G. R., \& Anchordoqui, L. A. 2015, PhRvD, 92, 123001

Verzi, V. 2019, PoS, ICRC2019, 450

Wanderman, D., \& Piran, T. 2010, MNRAS, 406, 1944

Wang, X.-Y., Liu, R.-Y., \& Aharonian, F. 2011, ApJ, 736, 112

Wang, X.-Y., Razzaque, S., \& Mészáros, P. 2008, ApJ, 677, 432

Watson, A. A. 2014, RPPh, 77, 036901

Webb, G. M., \& Gleeson, L. J. 1979, Ap\&SS, 60, 335

Yüksel, H., \& Kistler, M. D. 2007, PhRvD, 75, 083004

Yüksel, H., Kistler, M. D., Beacom, J. F., \& Hopkins, A. M. 2008, ApJL, 683, L5

Yushkov, A. 2019, PoS, ICRC2019, 482

Zatsepin, G. T., \& Kuz'min, V. A. 1966, JETPL, 4, 78 